\title{
The neuropathic potential of anti-GM1 autoantibodies is regulated by the local glycolipid environment in mice
}

\author{
Kay N. Greenshields, ${ }^{1}$ Susan K. Halstead, ${ }^{1}$ Femke M.P. Zitman, ${ }^{2,3}$ \\ Simon Rinaldi, ${ }^{1}$ Kathryn M. Brennan, ${ }^{1}$ Colin O'Leary, ${ }^{1}$ Luke H. Chamberlain, ${ }^{4}$ \\ Alistair Easton, ${ }^{1}$ Jennifer Roxburgh, ${ }^{1}$ John Pediani, ${ }^{4}$ Koichi Furukawa, ${ }^{5}$ \\ Keiko Furukawa, ${ }^{5}$ Carl S. Goodyear, ${ }^{1}$ Jaap J. Plomp, ${ }^{2,3}$ and Hugh J. Willison ${ }^{1}$
}

${ }^{1}$ Division of Clinical Neurosciences, Glasgow Biomedical Research Centre, University of Glasgow, Glasgow, United Kingdom. 2Department of Neurology and 3Department of Molecular Cell Biology — Group Neurophysiology, Leiden University Medical Centre, Leiden, The Netherlands. ${ }^{4}$ Henry Wellcome Laboratory of Cell Biology, Division of Biochemistry and Molecular Biology, Faculty of Biomedical and Life Sciences, University of Glasgow, Glasgow, United Kingdom. ${ }^{5}$ Department of Biochemistry II, Nagoya University School of Medicine, Nagoya, Japan.

\begin{abstract}
Anti-GM1 ganglioside autoantibodies are used as diagnostic markers for motor axonal peripheral neuropathies and are believed to be the primary mediators of such diseases. However, their ability to bind and exert pathogenic effects at neuronal membranes is highly inconsistent. Using human and mouse monoclonal antiGM1 antibodies to probe the GM1-rich motor nerve terminal membrane in mice, we here show that the antigenic oligosaccharide of GM1 in the live plasma membrane is cryptic, hidden on surface domains that become buried for a proportion of anti-GM1 antibodies due to a masking effect of neighboring gangliosides. The cryptic GM1 binding domain was exposed by sialidase treatment that liberated sialic acid from masking gangliosides including GD1a or by disruption of the live membrane by freezing or fixation. This cryptic behavior was also recapitulated in solid-phase immunoassays. These data show that certain anti-GM1 antibodies exert potent complement activation-mediated neuropathogenic effects, including morphological damage at living terminal motor axons, leading to a block of synaptic transmission. This occurred only when GM1 was topologically available for antibody binding, but not when GM1 was cryptic. This revised understanding of the complexities in ganglioside membrane topology provides a mechanistic account for wide variations in the neuropathic potential of anti-GM1 antibodies.
\end{abstract}

\section{Introduction}

The sialic acid-containing glycosphingolipids known as gangliosides are concentrated in plasma membrane microdomains, where they modulate the topological organization and function of membrane proteins $(1,2)$. Their oligosaccharide head groups protrude from the lipid bilayer into the extracellular environment to act as (co)receptors for a diverse range of glycan-binding proteins, including autoantibodies, sialic acid-binding Ig-like lectins (siglecs), microbial toxins, and viral components (3-8).

In a subset of autoimmune peripheral nerve diseases, including Guillain-Barré syndrome (GBS) and multifocal motor neuropathy, autoantibody-ganglioside interactions are believed to be a critical pathogenic factor $(9,10)$. Serum anti-GM1, -GD1b, -GQ1b, and -GD1a ganglioside antibodies are associated with nerve injury in both human clinical studies and animal models (11-14), with antiGM1 antibodies being highly associated with motor neuropathy variants (9). With respect to the antibody induction phase of the illness, it is clearly established that anti-GM1 antibodies can arise through molecular mimicry with structurally homologous Cam-

Conflict of interest: The authors have declared that no conflict of interest exists. Nonstandard abbreviations used: $\alpha B T x, \alpha$-bungarotoxin; CTB, cholera toxin B subunit; GBS, Guillain-Barré syndrome; Gal $(\beta 1-3)$ GalNAc, galactose- $\beta$ 1-3 $N$-acetylgalactosamine; GalNAcT, $N$-acetylgalactosamine transferase; GD3s, GD3 synthase; GPI, glycosylphosphatidylinositol; LOS, lipooligosaccharide; MAC, membrane attack complex; MEPP, miniature endplate potential; NHS, normal human serum; NMJ, neuromuscular junction; PI-PLC, phosphatidylinositol-specific PLC.

Citation for this article: J. Clin. Invest. 119:595-610 (2009). doi:10.1172/JCI37338. pylobacter jejuni lipooligosaccharides (LOS) (15-18). In contrast, examination of the effector pathways through which anti-GM1 antibodies selectively bind to and induce injury in motor nerve membranes, while avoiding damage to other neural and non-neural plasma membranes containing abundant GM1, is confounded by inconsistent and often counterintuitive data $(9,19-22)$. In particular, the sensitivity or resistance of the membrane toward undergoing anti-GM1 antibody-mediated injury cannot be fully explained by the presence and density of plasma membrane GM1.

One reason for the uncertainties surrounding anti-GM1 effector pathways may be that protein-ganglioside interactions are typically identified by in vitro solid-phase binding studies using immobilized gangliosides or structurally related natural and synthetic glycans. The translation of this in vitro binding data to physiologically and pathophysiologically relevant protein-glycan binding behavior in intact membranes in vivo is where the complexities and inconsistencies arise. For example, an antibody that binds a specific glycan by immunoassay may apparently be unable to bind the same ganglioside when present in an intact membrane (23). Furthermore, different anti-GM1 antibodies can have very different binding patterns in the CNS $(24,25)$. In addition to differences in antibody affinities, one explanation for such discrepancies might be that, within the complex environment of glycolipid-enriched microdomains, the interacting oligosaccharide headgroup is masked from the protein binding partner by surrounding molecules. Furthermore, fixation procedures might influence the antibody-binding characteristics of gangliosides 


\section{Table 1}

Mouse and human mAb ganglioside binding characteristics

\begin{tabular}{|c|c|c|c|c|c|c|c|c|}
\hline \multirow[t]{2}{*}{$\mathrm{mAb}$} & \multirow[t]{2}{*}{ Isotype } & \multirow[t]{2}{*}{ Immunogen } & \multicolumn{4}{|c|}{ Half-maximal binding ${ }^{A}$} & \multicolumn{2}{|c|}{ Affinity } \\
\hline & & & GM1 & GA1 & GD1b & GD1a & $K_{D}^{B}$ & Ligand \\
\hline \multicolumn{9}{|l|}{ Mouse } \\
\hline DG1 & $\lg G 2 b$ & HS19 LOSC (GM1+, GT1a+) & $2.0 \times 10^{3}$ & - & - & - & $1.8 \times 10^{-6}$ & GM1 \\
\hline DG2 & $\lg G 3$ & GM1 liposome & $2.5 \times 10^{3}$ & $6.7 \times 10^{2}$ & $2.0 \times 10^{2}$ & - & $3.5 \times 10^{-7}$ & GA1 \\
\hline MOG1 & $\lg G 3$ & GD1b liposome & - & - & $5.0 \times 10^{3}$ & & $1.0 \times 10^{-8}$ & GD1b \\
\hline MOG35 & $\lg G 2 b$ & 0:19 LOSC $\left(G M 1+\right.$, GD1a $\left.^{+}\right)$ & - & - & - & $1.0 \times 10^{4}$ & $9.5 \times 10^{-7}$ & GD1a \\
\hline \multicolumn{9}{|l|}{ HumanD } \\
\hline SM1 & $\lg M$ & Unknown & $6.5 \times 10^{3}$ & 50 & - & - & Unknown & \\
\hline D01 & $\lg M$ & Unknown & $5.5 \times 10^{3}$ & $4.3 \times 10^{3}$ & $5.7 \times 10^{3}$ & - & Unknown & \\
\hline
\end{tabular}

AHalf-maximal binding values represent the reciprocal of the antibody concentration (in $\mathrm{mg} / \mathrm{ml}$ ) giving half maximal binding as determined by ELISA. BBiaCore analysis of antibody Fab fragments was used to determine the binding affinity $\left(K_{D}\right)$ of the antibodies to the shown ligands (29). CHS19 and 0:19 are the $C$. jejuni strains from which the LOS species were isolated and used to immunize GalNAcT ${ }^{-1-}$ mice (15). ${ }^{\mathrm{D} C l o n e d}$ from human patients with acute and chronic motor neuropathy (38).

(23). However, the detailed mechanisms underlying these determinants of antibody-ganglioside binding are unknown.

In the current study, we addressed these issues by investigating a group of mouse and human anti-GM1 mAbs for their potential neuropathogenic effects at mouse motor nerve terminals and by studying in detail the underlying topological requirements for their binding to GM1 in neuronal membrane. Previously we showed that anti-GQ1b and anti-GD1a antibodies bind to the presynaptic motor nerve ending and activate complement, leading to membrane attack complex (MAC, C5b-9) formation, which causes intense neurotransmitter release and ultrastructural destruction, thereby blocking synaptic transmission at the neuromuscular junction (NMJ) $(11,14$, $26,27)$. We here show that mouse and human anti-GM1 mAbs also bring about these destructive neuropathic effects but that antigenic GM1 in the living neuronal membrane is cryptic for a proportion of the $\mathrm{mAbs}$, thereby rendering them harmless, due to a masking effect of neighboring gangliosides including GD1a. These findings indicate that anti-GM1 antibodies are pathogenically relevant in motor neuropathy and that masking and unmasking of GM1, such as may occur in membranes undergoing dynamic changes associated with normal axonal maintenance or during axonal regeneration, might have a profound influence on antibody pathogenicity (28).

\section{Results}

Anti-GM1 binding characteristics of mouse and human mAbs. The origin and properties of the anti-GM1 mouse mAbs DG1, DG2, antiGD1b mAb MOG1, and anti-GD1a mAb MOG35 and human anti-GM1 mAbs SM1 and DO1 (derived from patients with motor neuropathy) are shown in Table 1 . As revealed by ELISA, DG1 and DG2 react with GM1 with very similar half-maximal binding values $(0.5$ and $0.4 \mu \mathrm{g} / \mathrm{ml}$ respectively), as previously reported (29). Screening by ELISA against a panel of gangliosides (GM1, GM2, GM3, GD1a, GD1b, GT1a, GT1b, GQ1b, GD2, GD3, GA1) demonstrated that DG1 only binds GM1, suggesting a binding epitope unique to GM1 that includes the internal sialic acid, whereas DG2 binds GM1, GA1, and more weakly to GD1b, suggesting that the epitope principally comprises the nonreducing galactose- $\beta$ 1-3 $N$-acetylgalactosamine $[\mathrm{Gal}(\beta 1-3) \mathrm{GalNAc}]$ moiety common to the 3 glycolipids. Supplemental Figure 1 (supplemental material available online with this article; doi:10.1172/JCI37338DS1) shows details of relevant gangliosides and epitopes. Antibody sequencing has previously determined the variable region gene usage of DG1 and DG2 and established that they contain different immunoglobulin heavy and light chain gene rearrangements and thus have distinct clonal origins (29). Human mAb SM1 binds principally to GM1, and mAb DO1 binds to the Gal( $\beta 1-3)$ GalNAc moiety common to GM1, GA1, and GD1b.

Both DG1 and DG2 bind to motor nerve terminals in frozen nerve-muscle tissue. Prior to evaluating the ability of DG1 and DG2 to mediate any pathogenic effects on nerve, we first assessed the binding potency of DG1 and DG2 to NMJs in immunohistological analyses of unfixed frozen muscle-nerve preparation of diaphragm from WT, GD3 synthase-deficient (GD3s/-), and $N$-acetylgalactosamine transferase-deficient (GalNAcT ${ }^{-1}$ ) mice. The ganglioside composition of the glycosyltransferase-deficient mice is shown in Supplemental Figure 1. These mice are used because they are powerful tools for analyzing the relevance of specific ganglioside targets, with GM1 being absent in GalNAcT $T^{-/-}$mice and overrepresented, along with GD1a and other a-series gangliosides, in GD3s/mice, as previously reported (30). Frozen muscle sections were immunostained with DG1 or DG2 or, as positive control, with cholera toxin B subunit (CTB), a highly specific GM1 ligand (31). Fluorescence intensity and coverage of the NMJ area (defined by the area stained with fluorescent $\alpha$-bungarotoxin $[\alpha \mathrm{BT}$ ] $]$, labeling acetylcholine receptors at the NMJ) was quantitated. Under these conditions, all 3 ligands bound over nerve terminals in WT and GD3s/- tissue (Figure 1). The intensity of DG1 and DG2 immunostaining at NMJs was similar in $G D 3 s^{-1-}$ tissue $(\sim 100 \mathrm{AU})$, but the level was somewhat lower for DG1 than DG2 in WT tissue. As expected, in view of the GM1 deficiency, no DG1, DG2, or CTB staining was observed at NMJs of GalNAcT $T^{-1-}$ mice.

DG2 and DG1 have different capacities to bind to live mouse motor nerve terminals and to exert complement-mediated neuropathogenic effects. We next examined motor nerve terminal binding and possible pathophysiological effects of DG1 and DG2 at living NMJs in dissected triangularis sternae and diaphragm nerve-muscle preparations maintained as live preparations in oxygenated Ringer's medium. In positive control experiments conducted to demonstrate the presence and level of GM1 at nerve terminals, CTB bound strongly to both GD35/- (Figure 2A, left panel) and WT (data not shown) nerve terminals, with strong staining of the presynaptic axon of the NMJ. Since GM1 is widely distributed throughout the body, albeit at low 
A
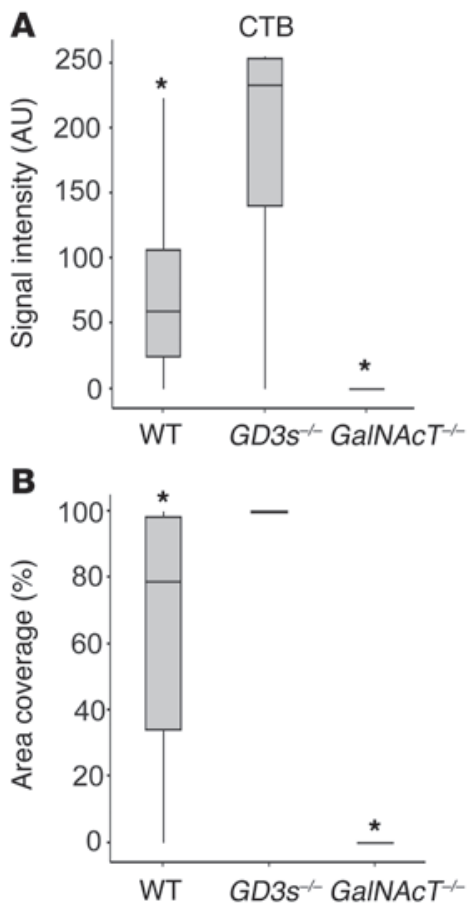

C
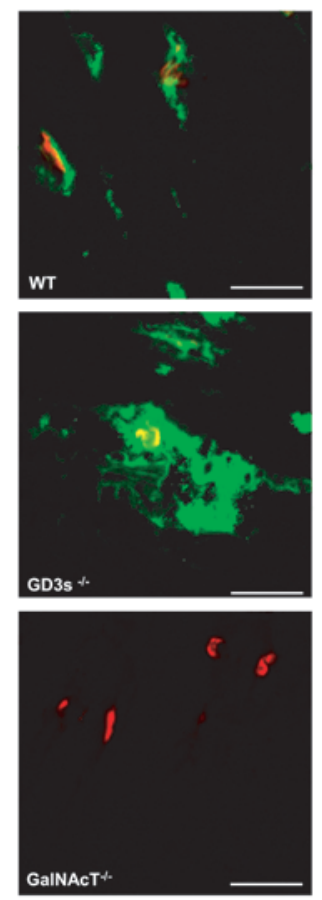

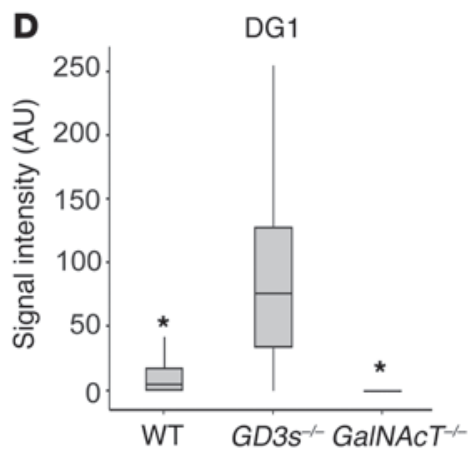

E

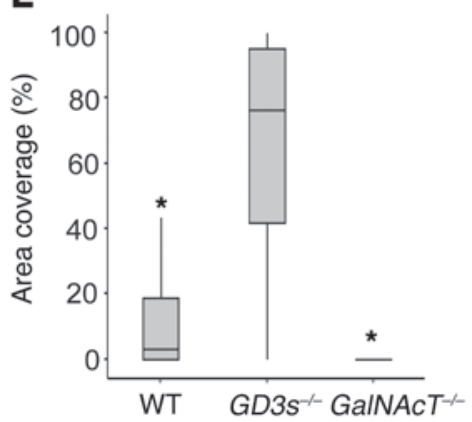

$\mathbf{F}$
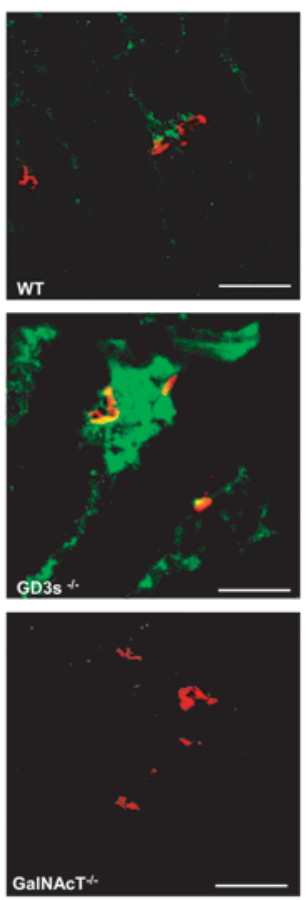

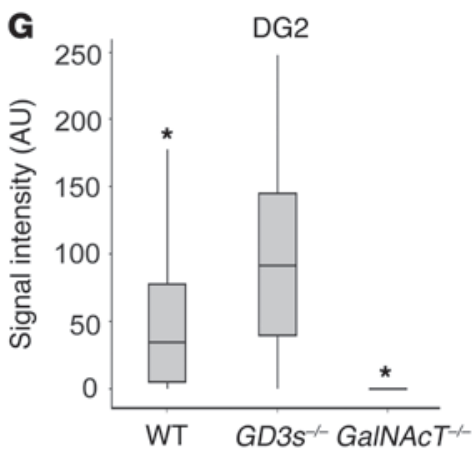

H

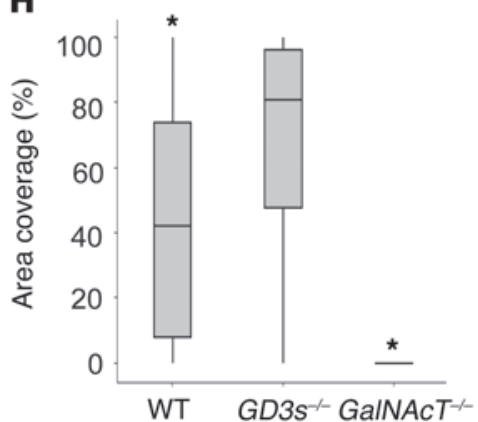

I
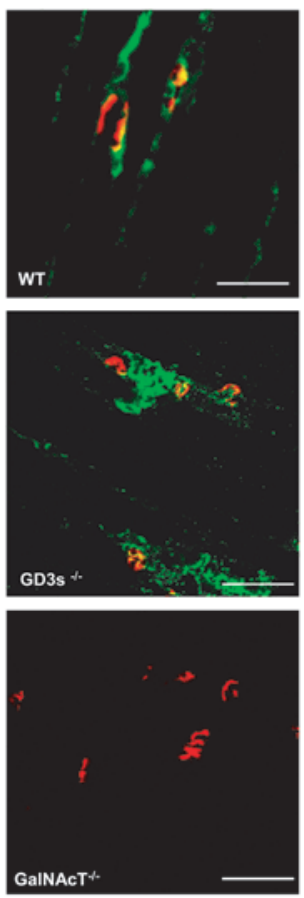

Figure 1

Topical immunostaining of frozen diaphragm sections from WT, GD3s ${ }^{-/}$, and GalNAcT $T^{-/-}$mice. Fluorescence was quantified as either the area of the NMJ (stained with $\alpha B$ Tx-TRITC) that is covered by anti-GM1 ligand (i.e., FITC fluorescence) or as the intensity of the FITC signal overlying the NMJ. All ligands bound most abundantly at the GD3s $s^{-1-}$ NMJ compared with the WT NMJ. ${ }^{*} P<0.05$, WT versus GD3s ${ }^{-1-}$. All images were acquired at $\times 40$ magnification. Scale bars: $60 \mu \mathrm{m}$. (A and B) CTB fluorescence is strong at the WT and GD3s ${ }^{-1-}$ but absent at the GalNAcT T- NMJs. (C) Examples of CTB binding at NMJs of each strain, taken under constant microscope settings to represent the

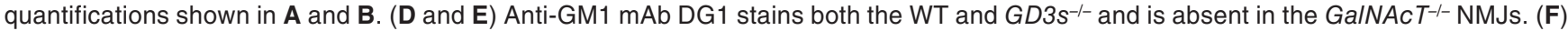
DG1 topical staining. (G and $\mathbf{H})$ DG2 binding profile is evident at WT and GD3s-/- but absent at the GalNAcT $T^{-/-}$NMJs. (I) DG2 binding at the WT, GD3s ${ }^{-/}$, and GalNAcT $T^{-/-}$NMJs. 
A
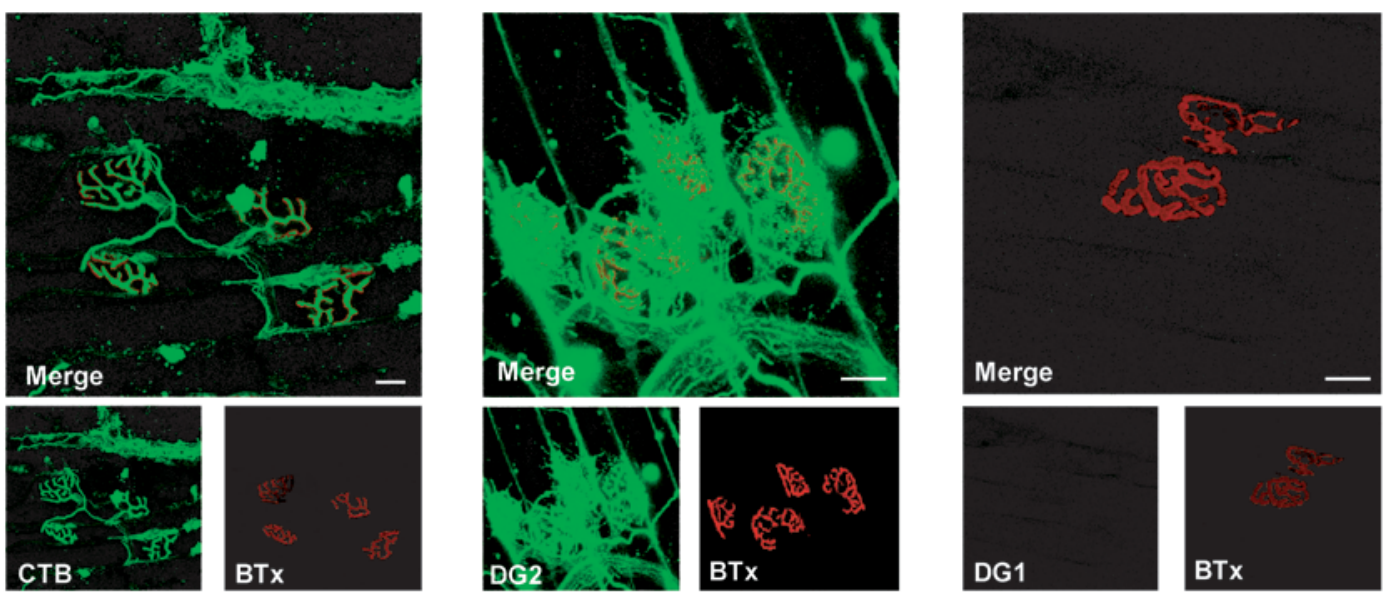

B
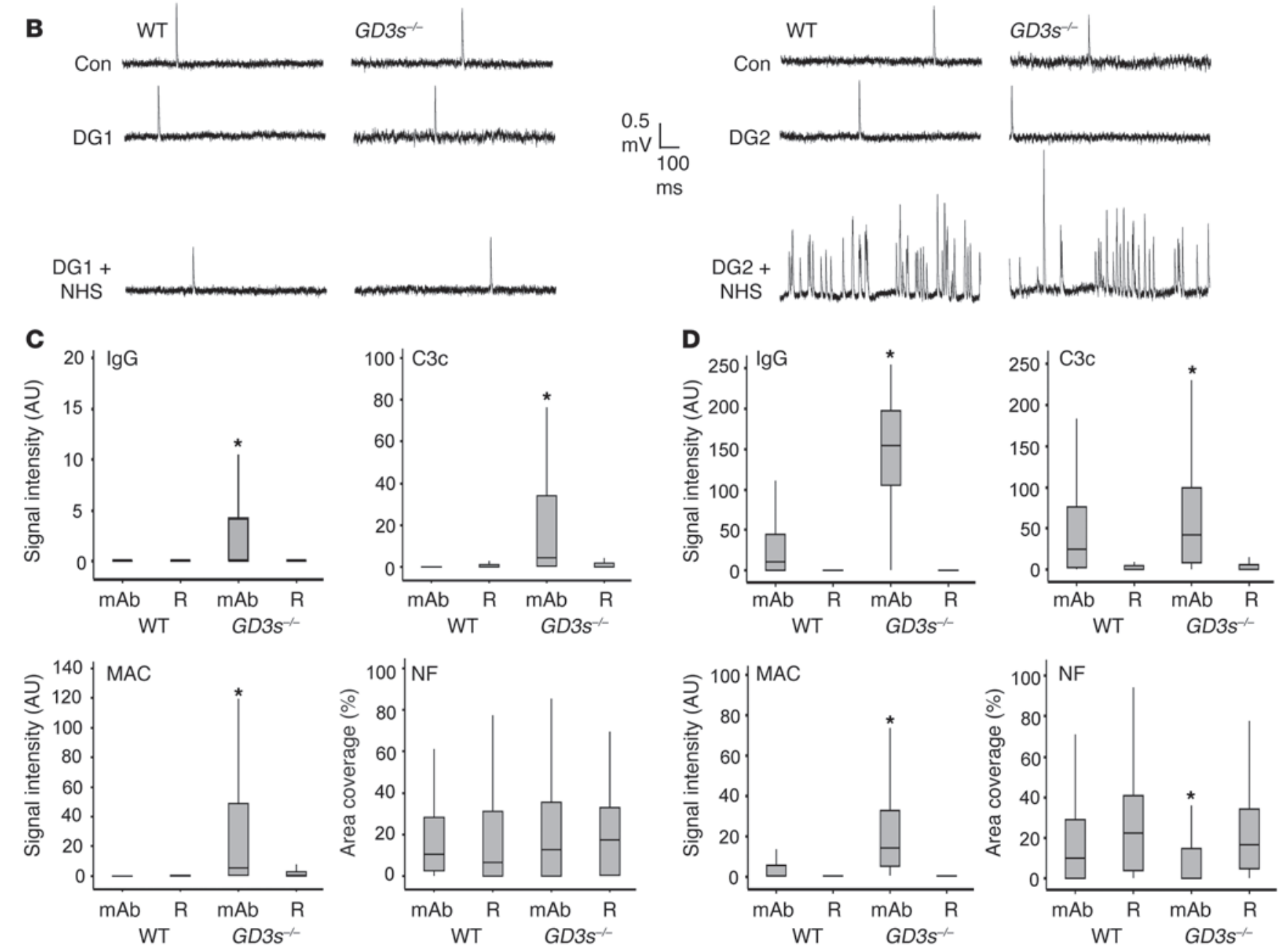


\section{Figure 2}

Pathophysiological effects of anti-GM1 antibodies in living nervemuscle preparations ex vivo. (A) Reconstructed confocal images of triangularis sterni NMJs from $\mathrm{GD} 3 \mathrm{~s}^{-/-}$mice, following ex vivo incubation in CTB, DG2, and DG1. For CTB and DG2, axonal staining is present along with staining of the parajunctional fibroblast. DG1 binding is undetectable. (B) Electrophysiology in ex vivo hemidiaphragm of WT and $G D 3 s^{-1-}$ mice. mAbs were applied, followed by a source of complement, and under such conditions only DG2 caused an increase in MEPP frequency of each strain. Con, control. (C) DG1 effect in ex vivo hemidiaphragm (WT and $G D 3 s^{-/}$). Following ex vivo muscle nerve incubations in mAb (or Ringer' s medium alone [R] as control), tissue was snap-frozen and sectioned for quantitative analysis. Graphs show IgG, C3c, and MAC deposition (quantified as the signal intensity over the NMJ). Neurofilament (NF) loss is quantified as area coverage over the NMJ. DG1 binding is undetectable in the WT NMJ and only weakly present in the $G D 3 s^{-1-} \mathrm{NMJ}$ under maximal detection settings. MAC and $\mathrm{C} 3 \mathrm{c}$ deposition do not translate into neurofilament loss. (D) The experiment was performed as described for $\mathbf{C}$, but with the mAb DG2. DG2 is detectable in WT and GD3s $s^{-1}$ NMJs and activates complement to cause a neurofilament loss compared with Ringer's medium alone-incubated control tissue. For both $\mathbf{C}$ and $\mathbf{D}$, note that the $y$ axes have been adjusted for clarity. ${ }^{*} P<0.05$ compared with the Ringer's control. Scale bars: $20 \mu \mathrm{m}$.

levels, staining was also seen elsewhere, including connective tissue and blood vessels; thus, CTB staining over other GM1-bearing structures is also seen in the upper region of Figure 2A (left panel). DG2 (Figure 2A, middle panel) showed a staining pattern similar to that of CTB, with particularly pronounced staining in this image of the parajunctional fibroblast, an extralaminar cell that caps the $\mathrm{NMJ}$ and also bears the GM1 ligand (32). GalNAcT $T^{-1}$ nerve terminals and other structures were not stained under these conditions,

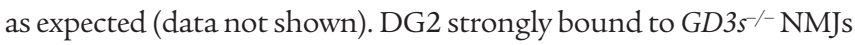
$(\sim 150 \mathrm{AU})$ and to a lesser extent (20-50 AU) to WT NMJs (Figure 2D). Unexpectedly, and in marked contrast to DG2 and to the situation observed in frozen sections as described above, DG1 binding was undetectable at all living WT NMJs, and DG1 bound only very weakly at GM1-rich GD3s/- NMJs (undetectable at $>50 \%$ of NMJs and only at $<10 \mathrm{AU}$ at the most strongly positive NMJs; Figure 2, A and C). As expected, neither of the $2 \mathrm{mAbs}$ bound at GalNAcT ${ }^{-1-}$ NMJs (Supplemental Figure 2A). Thus, whereas both DG1 and DG2 are able to bind well to GM1 at NMJs in frozen muscle-nerve preparation sections, only DG2 is capable of binding GM1 at live NMJs in physiological preparations, indicating that the topographical conformation of GM1 and/or adjacent molecules in the neuronal membrane is substantially different under these 2 conditions.

In order to study the ability of bound anti-GM1 antibody at live NMJs to activate complement and induce neuropathological effects, we exposed DG1- and DG2-pretreated live nerve-muscle diaphragm to normal human serum (NHS; as a complement source) and electrophysiologically monitored the occurrence of miniature endplate potentials (MEPPs, the spontaneous uniquantal acetylcholine release at NMJs) with intracellular microelectrode measurement. In the presence of human serum, DG2-pretreated NMJs showed a dramatic rise in MEPP frequency in WT and GD3s/preparations (Figure 2B and Supplemental Figure 2B), which caused asynchronous twitching of muscle fibers, which eventually subsided and was followed by complete synaptic "silence." This resulted in paralysis by block of transmission at the NMJ as judged from the inability of the diaphragm muscle to contract following phrenic nerve stimulation. As expected, in view of the finding that DG1 lacks binding activity to live WT NMJs, no such NHS-induced electrophysiological effects were observed in DG1pretreated preparations (Figure 2B and Supplemental Figure 2C). We screened for immunohistological evidence of antibodymediated complement activation by assessing $\mathrm{C} 3 \mathrm{c}$ and MAC deposition at NMJs (Figure 2, C and D, and Supplemental Figure 2). Both components were clearly deposited at $G D 3 s^{-1-}$ and WT nerve terminals in conjunction with DG2 binding, although with less intensity in WT. At DG1-pretreated nerve terminals from $G D 3 s^{-/}$mice in which very low levels of DG1 antibody were deposited, only very low levels of C3c (median, <5 AU) and MAC (median, <5 AU) were present. WT NMJs pretreated with DG1 showed no antibody binding and lacked any complement deposition. We next examined the structural integrity of the motor nerve terminal after MAC formation at the presynaptic membrane by quantifying neurofilament staining. DG2-pretreated NMJs from WT and GD3s/- mice that showed MAC deposition also displayed neurofilament loss (Figure 2D), whereas the very low level of DG1-dependent complement activation at GD3s/- NMJs was insufficient to induce such loss (Figure 2C).

Taken together, these results in living nerve-muscle preparations show that of the 2 anti-GM1 mAbs DG1 and DG2, only DG2 binds to motor nerve terminals and induces complement-mediated neuropathophysiological and structural effects at motor nerve terminals. Apparently, DG1 is inhibited from binding GM1, despite its abundance, by unknown factors in the local environment of the living neuronal membrane. The observed neuropathic effects of DG2 are identical to the complement-mediated effects we have seen previously for anti-GQ1b and anti-GD1a antibodies at NMJs $(11,14,33,34)$.

The nerve terminal ligand for DG1 and DG2 and the immunopathological target for DG2 in live membranes is GM1. The absence of nerve terminal staining in GalNAcT $T^{-1-}$ tissue with CTB, DG1, or DG2 (Figure 1) demonstrates that any potential ganglioside cross-reactivity of these GM1 ligands should only occur with the complex ganglioside series that are missing in GalNAcT ${ }^{-1-}$ mice (Supplemental Figure 1). However, in order to assess for certain whether DG1 and DG2 might be binding to any of these ganglioside antigens other than GM1 at the nerve terminal in either frozen or live tissue, we conducted blocking experiments using the GM1specific, high-affinity ligand CTB (Supplemental Figure 3A). In frozen tissue experiments, CTB pretreatment completely inhibited binding of DG1 and DG2 to GD $3 s^{-/-}$NMJs. Furthermore, CTB also inhibited DG2 binding in WT NMJs, excluding possible cross-reactivity with Gal( $\beta 1-3)$ GalNAc-bearing epitopes. We next demonstrated that DG2 (which binds GM1, GA1, and GD1b in ELISA) does not bind GD1b in the living neuronal membrane. We first showed the presence of GD1b at NMJs by incubating tissues with an anti-GD1b monospecific antibody, MOG1 (35), observing intense immunohistochemical staining at motor nerve terminals of WT mice (but neither GalNAcT $T^{-/-}$nor GD $35^{-1-}$ mice, which both lack GD1b). MOG1 also induced associated complement deposition and structural injury, as determined by neurofilament loss (Supplemental Figure 3B). We then showed that MOG1 binding was unaffected by CTB blockade (Supplemental Figure 3C). These data therefore exclude the possibility that DG2 is binding GD1b in the living tissue. They thus demonstrate that the Gal $(\beta 1-3)$ GalNAc epitope on GD1b is cryptically orientated for DG2 binding in vivo, whereas the MOG1-reactive GD1b epitope is available for MOG1 binding in vivo. Since GA1 (asialo-GM1) is absent from 
A
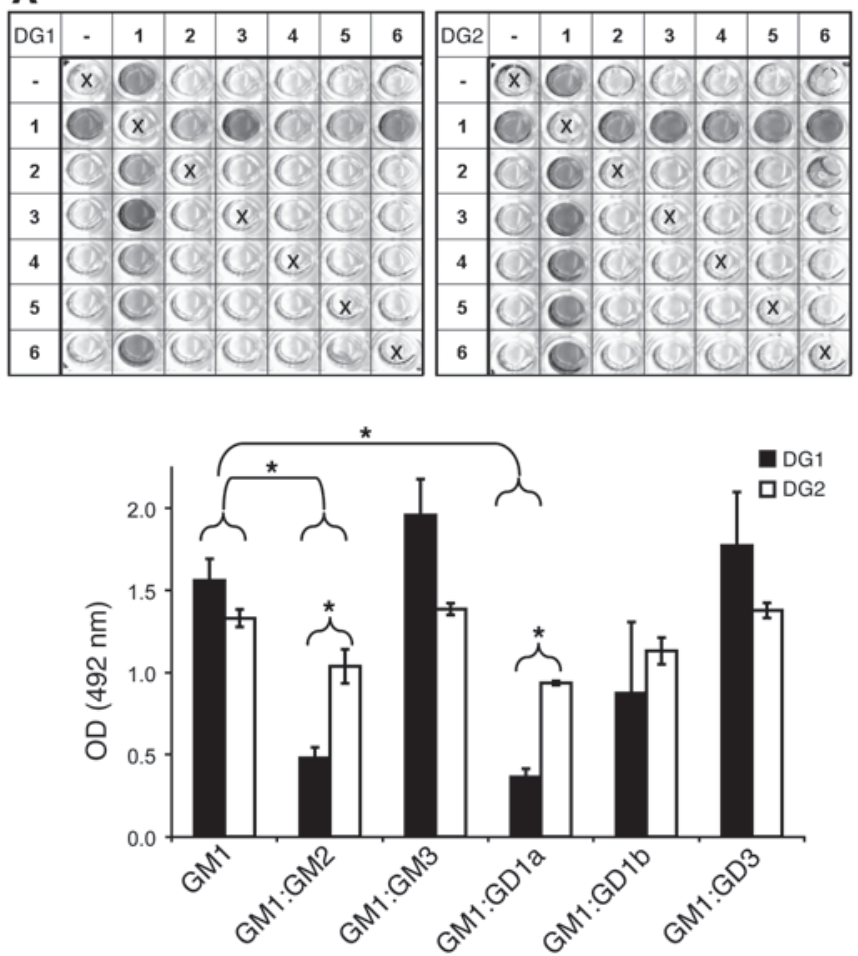

B

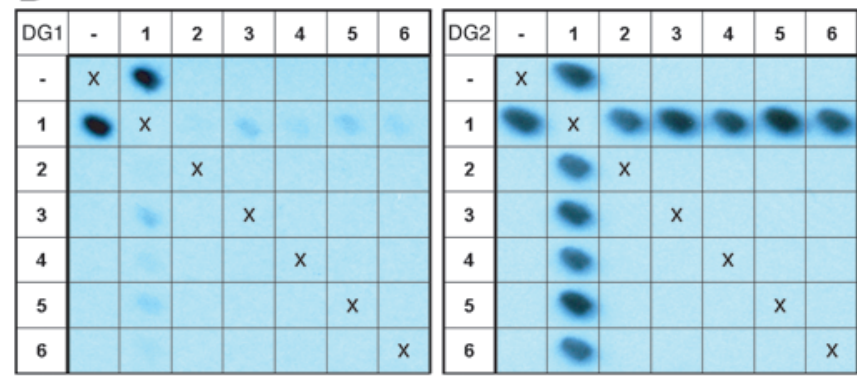

Figure 3

Reactivity of anti-GM1 mAbs DG1 and DG2 to ganglioside complexes containing GM1 in solid phase. The ganglioside complex at each location is established by combining the row and column labels. Thus, coordinates 1,4 and 4,1 represent GM1:GD1a complex. Wells labeled X are negative controls (methanol only). (A) ELISA. DG1 (left) and DG2 (right) both bind GM1 alone, with no difference in average OD. The binding of both antibodies to complexes of GM1 and GM2 or GD1a is reduced as compared with GM1 alone. No difference was observed with other combinations investigated (GM1:GM3, GM1:GD1b, and GM1:GD3). DG1 binding to complexes GM1:GM2 and GM1:GD1a was less than that of DG2. Mean results \pm SEM from 3 experiments are shown. (B) PVDF glycoarrays. DG1 was the primary antibody on the left membrane, DG2 on the right. Mean results \pm SEM for 3 experiments are shown. No significant difference in GM1 binding was observed for the 2 antibodies. DG1 binding to GM1 complexes was significantly reduced compared with GM1 alone $(P<0.05$, significance level for these comparisons not indicated in graph). DG2 binding GM1 complexes was marginally different compared with GM1 alone but significant for GM1:GM2 and GM1:GD1a. The inhibitory effect of complexes on antibody binding is greater for DG1 than for DG2. The average absolute reduction in signal intensity for GM1: GD1a complex, compared with GM1 alone, is $75.1 \%$ for DG1 and $18.2 \%$ for DG2 $(P<0.05)$. Error bars indicate SEM. 1, GM1; 2, GM2; 3, GM3; 4, GD1a; 5, GD1b; 6, GD3. ${ }^{*} P<0.05,{ }^{* \star} P<0.01$.

the mammalian nervous system (36), this potential cross-reactive antigen is not relevant in this context, and potential binding to $\mathrm{Gal}(\beta 1-3)$ GalNAc-bearing glycoproteins is excluded by the absence of staining in GalNAcT $T^{-1-}$ mice. Furthermore, and consistent with the complete blocking seen in frozen tissue sections, CTB preincubation of ex vivo muscle tissue from WT mice inhibits the complement-mediated actions of DG2, evidenced by abolition of C3c and MAC depositions over the NMJ (Supplemental Figure 3D). Collectively, these data indicate that DG2 is only binding GM1 in nerve terminal membranes in vivo. It should also be noted that the binding of DG2 to GD1b in ELISA is very weak (half-maximal binding, $2 \times 10^{2}$; see Table 1 ), further indicating that it is unlikely to bind avidly to GD1b in membranes.

Inbibitory interactions between GM1 and adjacent gangliosides form under solid-phase conditions. We speculated that the inability of DG1 to bind GM1 in the living plasma membrane was due to a masking effect arising from cis interactions with adjacent gangliosides and that these were disrupted following the death of tissue and subsequent processing to frozen sections. Since GD $3 s^{-/-}$mice are only able to synthesize a-series gangliosides, the most abundantly expressed species being GM1 and GD1a, we concluded that the masking effect in this mouse strain was most likely due to a GM1/GD1a cis interaction, notwithstanding the possibility that other gangliosides (e.g., GM2, GM3) could mediate similar blocking function in membranes with other ganglioside compositions. To investigate the potential inhibitory interaction between GM1 and GD1a for DG1 binding, we first attempted to recapitulate the in vivo interaction in solid-phase binding assays using ELISA and then using newly developed PVDF membrane formats (Figure 3). In ELISA, conducted with GM1:GD1a-coated at a 1:1 ratio by weight, DG1 was significantly inhibited and DG2 partially inhibited from binding GM1 in the presence of GD1a (Figure 3A). The mAbs were used at their GM1 half-maximal binding concentrations of $0.5 \mu \mathrm{g} / \mathrm{ml}$ and $0.4 \mu \mathrm{g} / \mathrm{ml}$, respectively. Furthermore, DG1 was significantly more impaired than DG2 from binding the GM1: GD1a complex. This inhibitory effect was not unique to GD1a, also occurring to a significant extent with GM1:GM2 and to a lesser extent (not significant) with GM1:GD1b. In marked contrast, complexes of GM1:GM3 and GM1:GD3 did not produce 
any inhibitory effects on DG1 or DG2 binding, which excludes the possibility that nonspecific displacement of GM1 by a second, nonreactive ganglioside accounts for the lower signal seen for the inhibitory GM1:ganglioside complexes.

When isolated GM1 was displayed on PVDF membranes as dot blots, there was no significant difference in the binding of DG1 and DG2 (Figure 3B). When GM1 was complexed with a range of other gangliosides, DG1 was almost completely inhibited from binding GM1. In contrast, DG2 binding to GM1 was minimally affected, although the difference in binding did reach significance for GM1:GM2 and GM1:GD1a complexes (Figure 3B). Throughout these PVDF studies, the reduction in complex binding for DG1 was substantially and significantly greater than that for DG2.

Under the PVDF array and ELISA assay conditions used in this study, DG2 is not demonstrated to bind to GD1b because of the very low half-maximal binding value for the DG2/GD1b interaction.

Inhibitory interactions between GM1 and GD1a are not present in C. jejuni LOS. To investigate whether this inhibitory interaction between GM1 and GD1a was a ganglioside-specific phenomenon or might also occur in other situations when GM1 and GD1a oligosaccharides were juxtaposed, we exploited the neuropathy-associated Campylobacter species that bear GM1 and GD1a mimics on their LOS (it should be noted that DG1 was cloned following immunization with LOS). A Penner O:19 C. jejuni isolate bearing both GM1 and GD1a mimics (50:50 ratio) in the core oligosaccharide was assessed for binding to DG1 (anti-GM1) and MOG35 (anti-GD1a) mAbs and CTB by FACS, in comparison with a nonganglioside-mimicking O:3 C. jejuni strain. DG1 was able to bind well to both live and killed (by UV light or paraformaldehyde) O:19 isolate, indicating that an inhibitory complex between the GM1- and GD1a-mimicking moieties of LOS is not present (Supplemental Figure 4). MOG35 and CTB also bound well to the O:19 isolate, confirming the presence of both the GM1 and GD1a mimics, and no ligands bound the control O:3 isolate (data shown for CTB only).

Access of DG1 to GM1 in the living membrane is prevented by a masking effect of GD1a. Based on the above data, we considered that the inability of DG1 to bind GM1 on the living neuronal membrane was due to closely adjacent gangliosides, including GD1a, which might cis-interact with GM1 to create a masking effect. Using live

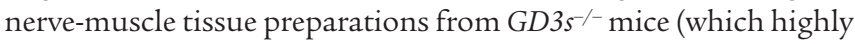
overexpress GM1 and GD1a), we cleaved the terminal sialic acid from GD1a with neuraminidase treatment, thereby converting GD1a to GM1, and subsequently assessed the live tissue for DG1 binding (Figure 4). This pretreatment massively promoted DG1 binding at the NMJ (Figure 4, A and B), which subsequently activated complement, as shown by $\mathrm{C} 3 \mathrm{c}$ and MAC deposition (Figure 4B). It thereby induced the pathophysiological cascade (Figure 4C), characterized by increased MEPP frequency, muscle fiber twitching, and paralysis, described above for DG2. This unmasking effect for DG1 was also evident in neuraminidase-treated tissue from $\mathrm{WT}$ C57BL/6 and BALB/c mice (Supplemental Figure 5A).

In control experiments, we verified that neuraminidase treatment was able to digest most of the GD1a present at motor nerve terminals, as indicated by a substantial reduction in the binding of the anti-GD1a mAb MOG35 after treatment, in comparison with strong binding before treatment (Supplemental Figure 5B). DG1 binding following neuraminidase treatment was also completely blocked by preincubation with CTB, indicating that binding of DG1 was GM1 specific (Supplemental Figure 5C). Since neur- aminidase treatment creates de novo GM1 from GD1a, it is very likely that this de novo GM1 would also be capable of acting as a ligand for DG1. Such binding confounds (without contradicting) the conclusion that masked "native" GM1 is released from its cis inhibition with GD1a by neuraminidase treatment to become available for DG1 binding. In order to investigate the extent to which the DG1 binding was directed to de novo GM1 generated from GD1a (rather than the preexisting, native GM1), we first blocked native GM1 in the tissue preparation with CTB and then treated the tissue with neuraminidase, followed by probing with DG1 (Supplemental Figure 5D). Under these conditions, DG1 binding to CTB-pretreated tissue was greatly reduced compared with binding to CTB-untreated tissue, indicating that a proportion of DG1 binding was to native GM1, unmasked from GD1a by neuraminidase treatment. After neuraminidase incubation, DG2 bound equally to both CTB-pretreated and -untreated tissue, indicating that abundant de novo GM1 produced from GD1a was available for DG2 binding. The relatively weak binding of DG1, in comparison with DG2 (Supplemental Figure 5D), to de novo GM1 (i.e., CTB-blocked, neuraminidase-treated tissue; Supplemental Figure 5D) suggests that not all the de novo GM1 is available for DG1 binding. One explanation for this would be that de novo GM1 might itself form inhibitory cis interactions with remaining a-series gangliosides (e.g., GM2, GM3) in the plasma membrane of $G D 3 s^{-1-}$ mice, although this was not addressed experimentally beyond the solid-phase PVDF membrane data shown in Figure 3.

GM1 and GD1 a colocalize to form an inbibitory environment for DG1 antibody binding in living PC12 cells and in lipid rafts. To investigate the molecular environment that might account for the masking effects of GD1a, we used rat neuroendocrine PC12 cells, which, in their undifferentiated state, express gangliosides including GM1 and GD1a (37). DG1 was entirely unable to bind GM1 in living PC12 cells, in contrast to strong binding with CTB, DG2, and the anti-GD1a mAb MOG35 (Figure 5), as predicted from the live nerve terminal findings. Neuraminidase treatment resulted in intense DG1 binding to PC12 cells, with complete concomitant loss of MOG35 binding (Figure 5B) under conditions in which cell viability was maintained, as monitored by ethidium homodimer-1 staining (data not shown). In colocalization studies of GM1 and GD1a, performed by comparing the binding of the mAbs DG2 and MOG35, the pixel-by-pixel correlation coefficient was 0.96 , indicating very close proximity at the light microscopy level (Figure 5C). These findings in PC12 cells indicate a PC12 membrane interaction between GM1 and GD1a similar to that occurring in the mouse motor nerve terminal membrane and thereby suggest that this cis-inhibitory behavior may be common in other plasma membranes.

Since gangliosides are enriched in lipid rafts (2), we considered that the raft microenvironment might favor interactions between GM1 and GD1a that were inhibitory for DG1 binding. First we disrupted rafts in live PC12 cells with the cholesterol-sequestering agent methyl- $\beta$-cyclodextrin to establish whether GM1 became available for DG1 binding. This was not observed; indeed, a general attenuation of ligand binding was seen (Supplemental Figure 6A). We next considered that raft-localized glycosylphosphatidylinositol-anchored (GPI-anchored) proteins might exert a masking effect on GM1 for DG1 binding and investigated this by cleaving them with phosphatidylinositol-specific PLC (PI-PLC). PI-PLC treatment enhanced DG2 and MOG35 binding, suggesting that GPI-anchored proteins can exert a general and nonspe- 
A
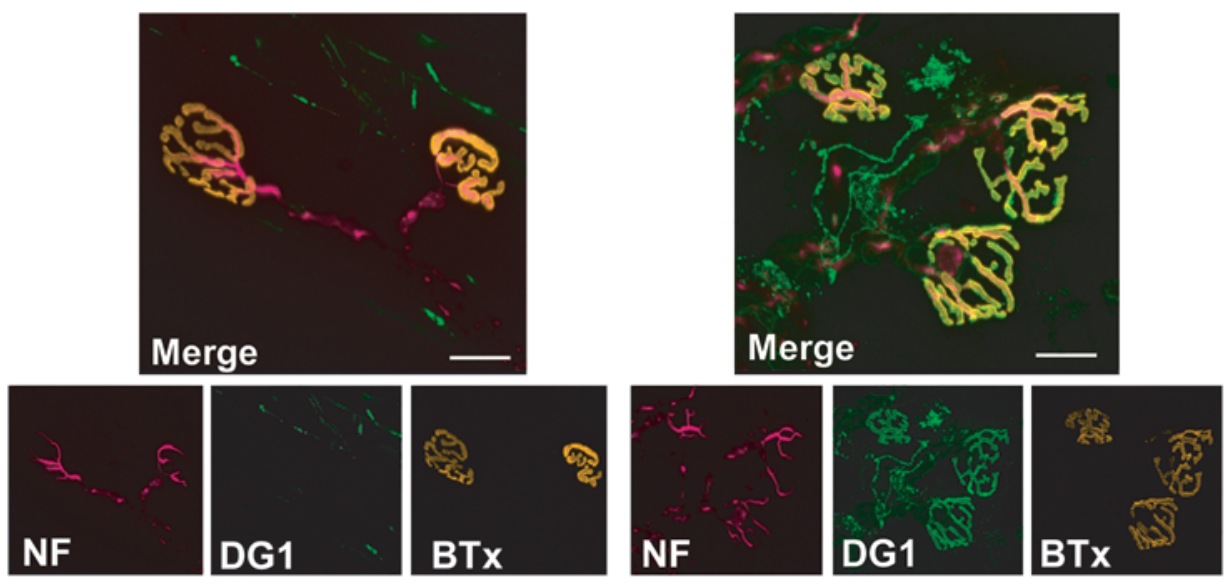

B
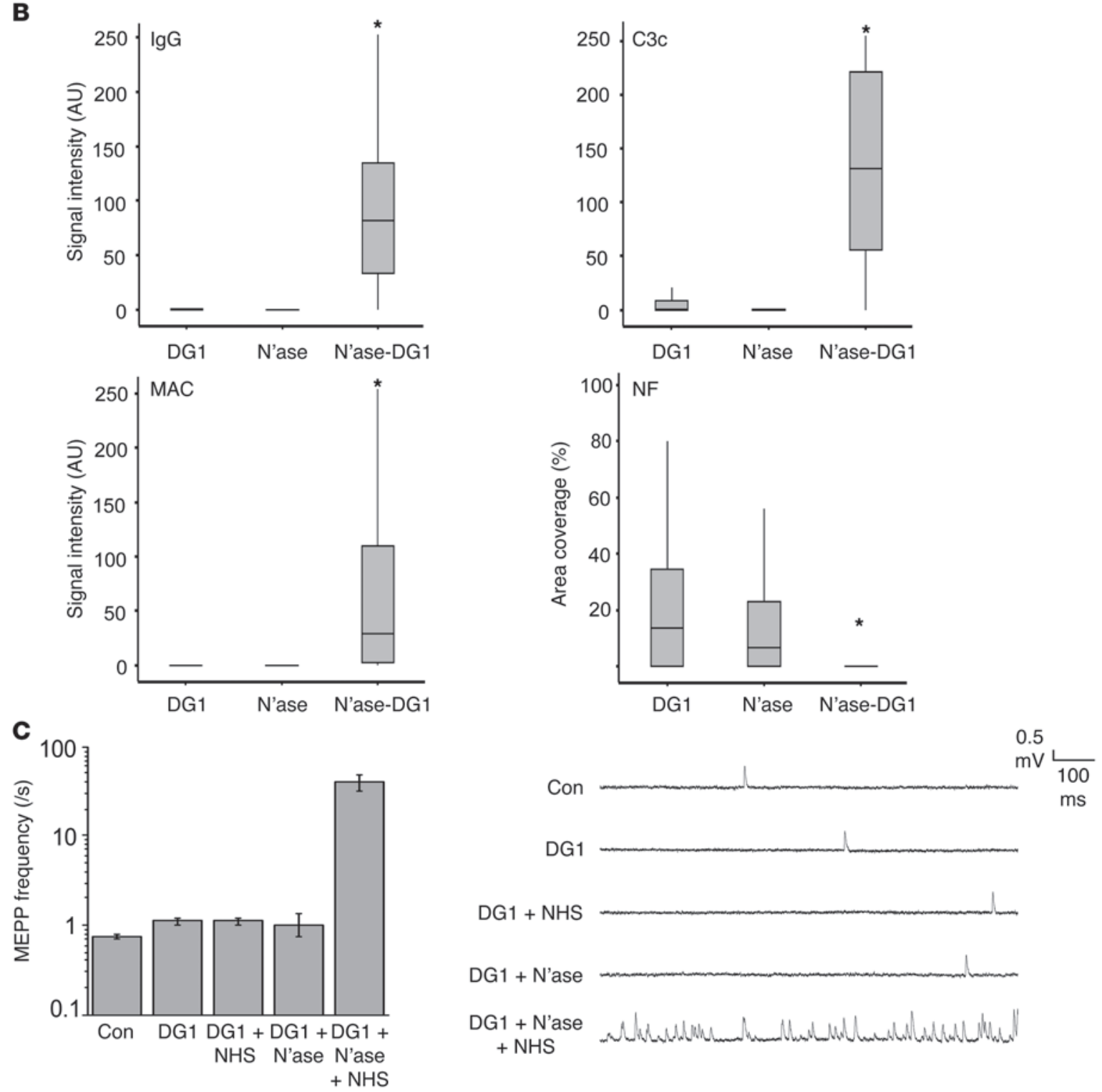

$$
\text { DG1 + NHS }
$$

DG1 + N'ase

DG1 + N'ase

$+\mathrm{NHS}$ 


\section{Figure 4}

Effect of neuraminidase treatment on anti-GM1 antibody binding and pathogenic activity. (A) Reconstructed confocal images of DG1 binding at ex vivo $G D 3 s^{-/-}$triangularis sterni NMJs. Left: DG1 binding in living tissue is undetectable at the NMJ (stained with $\alpha B T x-T R I T C$ ). Right: DG1 binding (FITC) following neuraminidase treatment of tissue. DG1 binding overlies the NMJ and is colocalized with the axonal neurofilament staining (Cy5), suggesting that DG1 is binding to the presynaptic aspect (the axon terminal). (B) Ex vivo $G D 3 s^{-1-}$ hemidiaphragm preparations, as described in Figure 2. Neuraminidase (N'ase) treatment of tissue prior to incubation with DG1 enabled DG1 to bind the NMJ (as shown by IgG deposition), fix complement, and cause a neurofilament loss. ${ }^{*} P<0.05$ compared with control tissue incubated in Ringer's (minus neuraminidase) followed by DG1. (C) Electrophysiology. DG1 and NHS as a source of complement caused a massive increase in MEPP frequency in the ex vivo $G D 3 s^{-1-} \mathrm{NMJ}$ only when applied to neuraminidase-pretreated tissue. Scale bars: $20 \mu \mathrm{m}$. Error bars represent SEM for experiments performed in triplicate.

cific masking effect on both GM1 and GD1a, but had no significant effect on DG1 binding, which remained negligible or absent (Supplemental Figure 6B). We also conducted the PI-PLC study at live NMJs and observed the same trend as seen in PC12 cells (Supplemental Figure 6C).

In order to support the hypothesis involving a molecular interaction between GM1 and GD1a, it would be necessary to show at least that they colocalize in the same raft environment. To investigate this, we incubated PC12 cells with the anti-GD1a mAb MOG35 and an irrelevant isotype control, EG1 (which reacts with GQ1b, GT1a, and GD3 but does not bind to PC12 cells; data not shown), and examined GD1a affinity-purified raft fractions by Western blot analysis for GM1 content (Figure 5D). This demonstrated that at least a proportion of GM1 was present in the GD1a-enriched raft compartment. In control studies, EG1 affinity purification of raft fractions did not isolate any raft material (as assessed by raft protein markers) or yield any GM1 or GD1a signal. The raft fraction containing GD1a and GM1 also contained the raft marker flotillin, whereas SNAP25 was undetectable, indicating that a heterogeneous population of rafts exist with respect to protein and ganglioside composition in PC12 cells. This latter point was not investigated further.

Human neuropathy-associated anti-GM1 mAbs also exert complement-mediated neuropathophysiological effects at the mouse motor nerve terminal, provided GM1 is unmasked. In order to bring clinical relevance to the findings observed with mouse mAbs, we screened human anti-GM1 antibodies cloned from motor neuropathy cases for similar behavior. Human anti-GM1 IgM antibodies SM1 and DO1 (38) were examined for their binding and complement-mediated neuropathophysiological effects at the mouse NMJ, and we assessed a possible inhibitory effect of interaction between GM1 and GD1a (Figure 6). Neither mAb bound to GD3s $s^{-1-}$ motor nerve terminals, and consequently they did not induce complement deposition (C3c and MAC) or neurofilament loss, as assessed by immunohistochemistry, and did not cause increased MEPP frequency, the electrophysiological hallmark of neuropathogenicity. However, following neuraminidase pretreatment of the nerve-muscle preparations, deposits of IgM were abundant. Consequently, the complement cascade was activated, as shown by $\mathrm{C} 3$ and MAC deposits, with resultant neurofilament loss and electrophysiological injury as manifested by an increased MEPP frequency.

\section{Discussion}

This study has 2 principal findings. First, we demonstrate that under specific conditions, both human and mouse anti-GM1 antibodies have potent complement activation-mediated neuropathogenic effects at mouse terminal motor axons, including electrophysiological and morphological injury, leading to block of synaptic transmission at the NMJ, thereby resulting in paralysis of the nerve-muscle preparation. Second, we discovered that GM1 ganglioside in living neuronal membranes is masked from binding certain classes of anti-GM1 autoantibody by an interaction with GD1a (and presumably other gangliosides) that is able to shield a critical domain of the oligosaccharide head group involved in antibody binding. Consequently, this masking prevented the neuropathophysiological effects of these anti-GM1 antibodies from occurring. These findings have major implications for our understanding of the pathogenic potential of anti-ganglioside antibodies in neuropathy, their use and relevance as autoimmune disease diagnostics, and as ganglioside detection reagents. The epitope masking effect of this interaction is not a feature concerning all anti-GM1 antibodies but is dependent upon subtle features of the antibody-antigen interaction that confer these characteristics and needs to be assessed for each antibody on a case-by-case basis. Importantly, our results demonstrate that the ability of mouse and human anti-GM1 antibodies to bind isolated GM1 in solid-phase assays or in frozen tissue sections cannot be used to predict their pathogenic behavior in vivo. Our findings are likely to account for the markedly discrepant effects of anti-GM1 antibodies observed in many previously published models.

Anti-GM1 antibodies are mainly associated with the acute motor axonal variants of GBS and chronic multifocal motor neuropathies (9), and our immunohistochemical and electrophysiological findings at motor nerve terminals may provide a neuropathophysiological explanation for a component of the paralytic symptoms in such patients. In support of this as a clinically relevant site, motor nerve terminal degeneration has been shown at NMJs in a motor point muscle biopsy of an acute motor axonal neuropathy variant GBS patient with anti-GM1 antibodies, while sural nerve biopsy was normal (39). The terminal portion of motor axons may be relatively vulnerable for autoimmune attack because it is not protected by a blood-nerve barrier, in contrast to more proximal parts of the axon. GM1 is also present at nodes of Ranvier throughout the length of the nerve. Our results certainly do not exclude antiGM1 binding and complement-mediated neuropathogenic effects at the more proximal parts of the axon, as has been observed in a rabbit neuropathy model (40).

The complement-mediated pathogenic effects of anti-GM1 mAbs at the NMJ shown here are identical to those observed by us in earlier (ex vivo and in vivo) mouse studies on mAbs against GQ1b ganglioside and on anti-GQ1b-positive patient sera (14, 27, 34). Furthermore, anti-GD1a antibodies produce these effects, provided the density of GD1a is high, as in neuronal membranes of GD3s/- mice (11). Thus, this neuropathophysiological effect at murine NMJs is a common feature of certain classes of anti-GM1, -GD1b, -GQ1b, and-GD1a antibodies. The heterogeneous distribution of clinical effects observed in patients with these anti-ganglioside antibody specificities does not correlate with the common paralytic effect of these antibodies at mouse NMJs. It is thus likely that regional differences in ganglioside expression, coexpression, and density in different neural and glial membranes from different anatomical regions in different species are critical determining factors. 
A
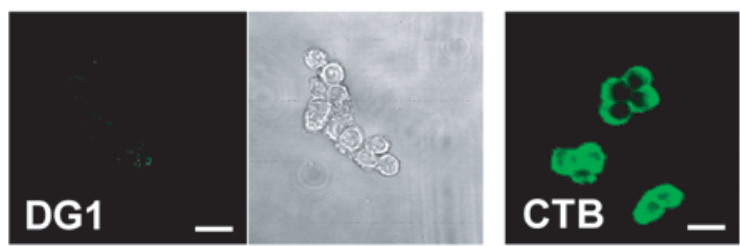

B
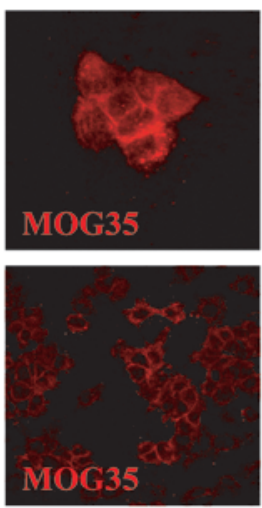

\section{DG1}
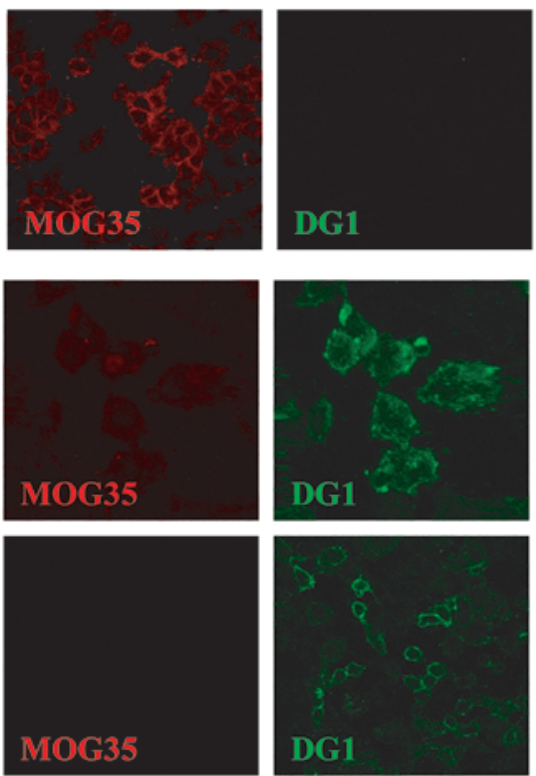

C
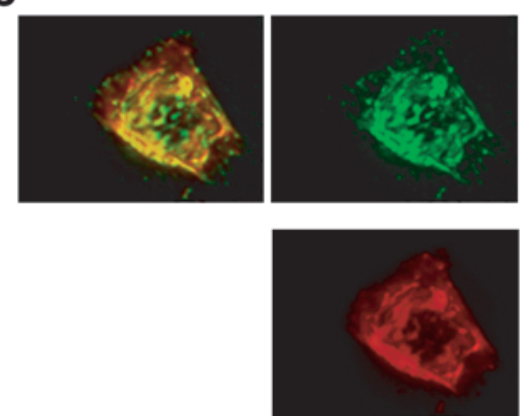
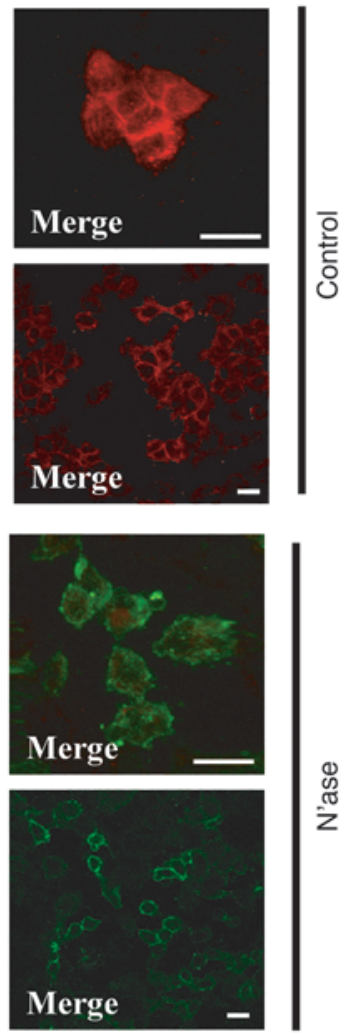

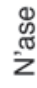

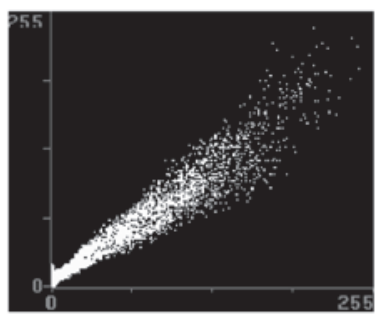

D Control mAb MOG35

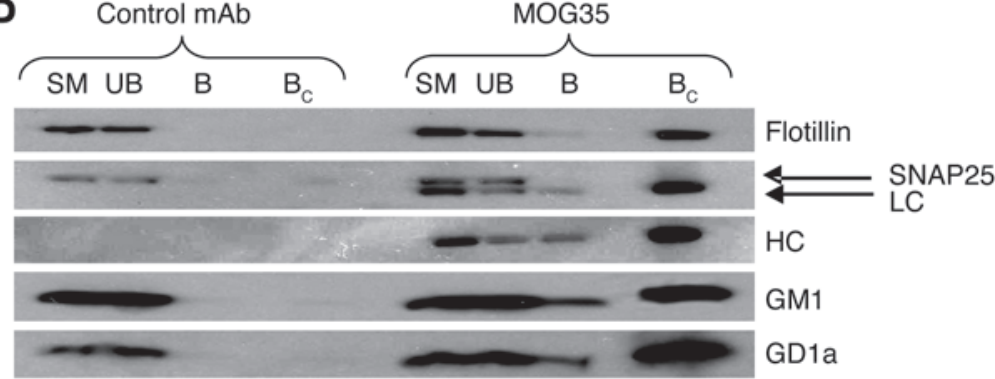

\section{Figure 5}

Localization of GM1 and GD1a to raft fractions in PC12 cells. (A) PC12 cell immunostaining (original magnification, $\times 40$ ). DG1 binding to PC12 cells (shown in phase contrast) is not detectable despite the presence of GM1, as shown by CTB staining of cells. Scale bars: $15 \mu \mathrm{m}$. (B) Effect of neuraminidase on DG1 and MOG35 binding to PC12 cells. Double staining reveals that control cells are positively stained with MOG35 (TRITC), with no binding of DG1 (FITC). Following neuraminidase treatment, MOG35 staining is diminished, with a concomitant increase in DG1 binding. Scale bars: $15 \mu \mathrm{m}$ (images acquired at $\times 40$ magnification). (C) GM1 and GD1a pixel-by-pixel colocalization. FITC and TRITC (anti-GM1 and anti-GD1a, respectively) images ( $\times 63$ magnification) from double-stained PC12 cells, with colocalization appearing as yellow overlap. Plane-by-plane colocalization (linear scatter plot) shows strong colocalization. (D) Western blot of raft immunoprecipitation based on MOG35 binding, allowing isolation of GD1a-positive rafts by anti-mouse IgG-coated beads. In irrelevant antibody-incubated cells, no rafts were isolated by anti-mouse IgG-coated beads. Bound sample was concentrated $(\times 10)$ to amplify any potentially weak signal. In MOG35-incubated cells, a population of rafts was isolated by the beads. Isolated fractions contained the raft-associated protein flotillin, but not SNAP25, which was taken as evidence that the raft extraction procedure did not lead to coalescence of the heterogeneous raft population. Bound fractions also contained both the light chain (LC) and heavy chain (HC) of the anti-GD1a antibody, and the isolated rafts were positive for both GM1 and GD1a. SM, starting material; UB, unbound; B, bound; $\mathrm{B}_{\mathrm{C}}$, bound concentrated. 
A
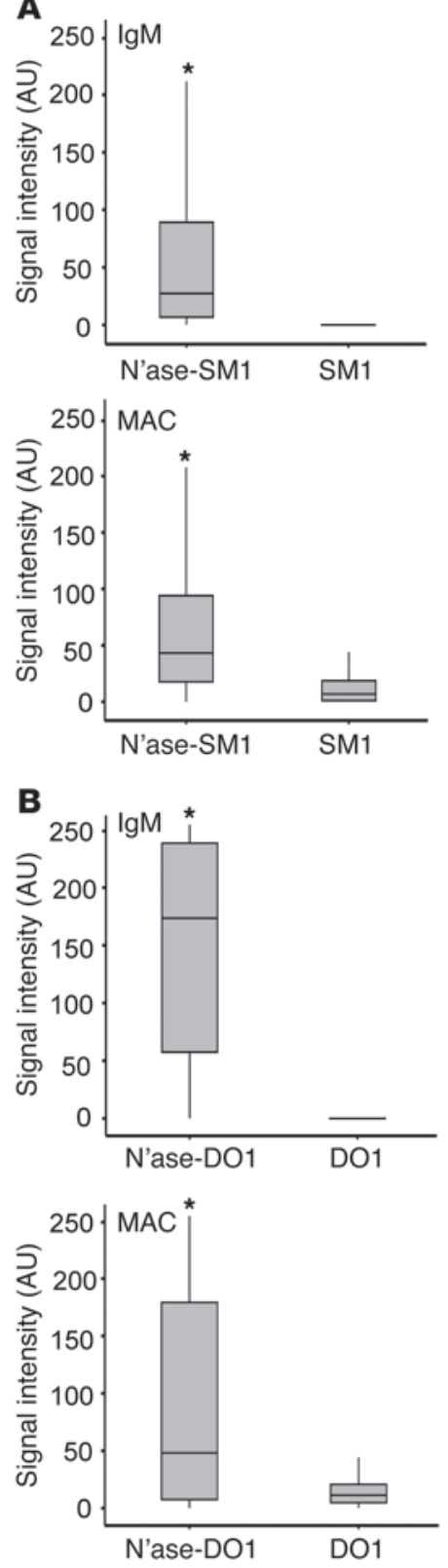
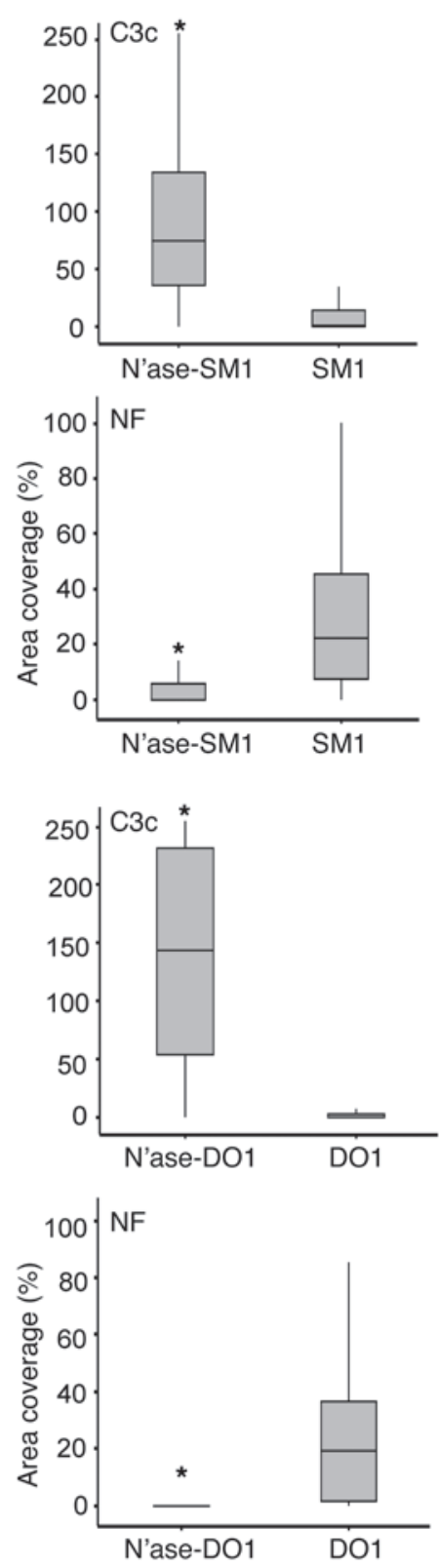

C
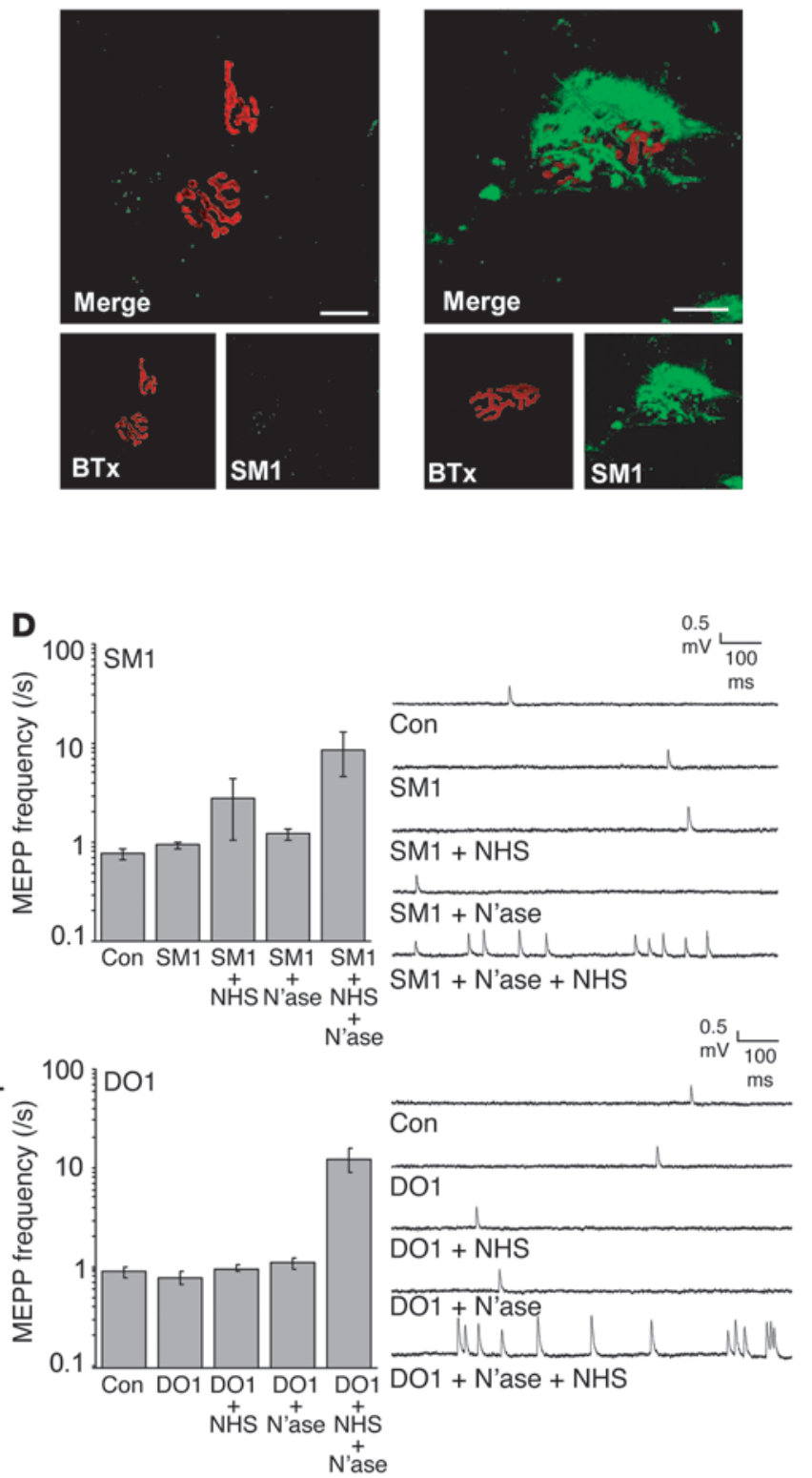

Figure 6

Binding and neuropathophysiological effects of human anti-GM1 mAbs SM1 and DO1. (A) Ex vivo hemidiaphragm preparations from GD3s ${ }^{-/}$ mice, as described for Figure 2. SM1 only binds NMJs following neuraminidase treatment and then causes a complement-mediated neurofilament loss. (B) The experiment was performed as described for A, but with DO1. (C) Absence of SM1 staining at GD3s $s^{-/}$triangularis sterni NMJs (left), and presence of staining when the tissue was preincubated in neuraminidase (right). (D) Electrophysiological analysis of the effects of SM1 and DO1 at GD3s ${ }^{-/}$hemidiaphragm NMJs, as described in Figure 4 for mouse mAb DG1. Following neuraminidase treatment, both SM1 and DO1 bind at NMJs and cause a complement-dependent increase in MEPP frequency. Scale bars: $20 \mu \mathrm{m}$. Error bars represent SEM for experiments performed in triplicate.

The differences in binding and pathogenic properties of individual anti-glycolipid antibodies may in part be attributable to differences in their affinity or avidity, in addition to their precise specificity. If the affinity of an antibody is high enough to compete effectively for a critical oligosaccharide domain that may otherwise be masked by complex formation with a cis-interacting glycolipid, then the pathophysiological consequence of any complex formation might be annulled. Whether this range of affinities can be achieved for anti-glycolipid antibodies is unknown. However, in the case of the widely used high-affinity GM1 ligand CTB, the affinity for GM1 is reported as being in the $10^{-10}$ to $10^{-12} \mathrm{M}$ range (41), which is $10^{4}$ - to $10^{6}$-fold higher than that of the mAb DG1, used in the current study. Structural studies have demonstrated that CTB binds GM1 through a 2-fingered pinch interaction involving the nonreducing terminal galactose epitope of GM1 and the internal sialic acid, the latter being masked in our complex for- 
mation model (41). DG1 also requires the presence of this internal sialic acid for binding to GM1. The implication is therefore that the very high affinity of CTB for GM1 is sufficient to disrupt an inhibitory complex that disguises the sialic acid from its binding domain on the toxin. In contrast, the affinity of DG1 for GM1 is substantially lower than that of CTB, which most likely accounts for DG1's inability to compete effectively for the cryptic sialic acid. Thus, affinity may be a relevant factor in disrupting glycolipidglycolipid complex interactions to allow ligand binding in certain situations. The affinities of DG2 and DG1 for GM1 and GA1 were within 5-fold of each other, and the half-maximal binding values for GM1 were very similar, yet they were completely polarized in terms of their pathogenic potential. It is therefore improbable that a simple explanation of affinity or avidity differences, regardless of dependence upon any complex formation, is the critical factor influencing binding in the current setting but more likely that the pathogenic differences are due to differences in the fine specificity of the 2 antibodies, specifically their dependence upon the internal sialic acid for binding. Other experimental evidence discussed below also strongly supports this view.

Our study underscores the complexities surrounding the topographical organization of glycolipids in living membranes and how these relate to topography in other types of detection systems such as are used in laboratory immunoassays. Considerable effort is currently focused on achieving optimal platforms for assessing protein-glycan interactions, and the issues surrounding complex formation need to be considered in this context (42). The cryptic behavior of glycolipid headgroups in the plasma membrane, as assessed by accessibility to antibodies, is a long-recognized phenomenon $(43,44)$, but its structural basis has, to our knowledge, never been demonstrated experimentally. This behavior may in part be dependent upon ganglioside organization in lipid rafts. In this study, raft disruption with a cholesterol-sequestering agent did not allow the DG1 antibody to bind GM1, indicating that the interaction of GM1 and GD1a is not cholesterol dependent, as we also observed by solid-phase immunoassay. Rather, it appears to be an intrinsic property of the interacting ganglioside species, possibly localized in a glycosphingolipid signaling subdomain (45). The ganglioside clustering property may be due to the glycosphingolipid hydroxyl groups acting as hydrogen bond donors, allowing side-by-side interactions within a microdomain (46). However, gangliosides require substantial surface area to host their oligosaccharide chain and its associated hydration water, implying that complex headgroups such as GM1 and GD1a will have large space requirements in the membrane (47), the consequence of which is unknown in terms of influencing their cis interaction.

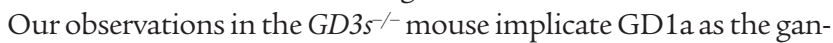
glioside that masks GM1, because in this artificial context, these are the 2 predominant gangliosides. Our data on WT mice and in solidphase assays also strongly suggest that other gangliosides (but not necessarily all gangliosides) may be capable of mediating this masking effect. In the GD35/- mouse, the GM1 concentration is substantially higher than in WT mouse, thereby adding support to the view that the failure of DG1 to bind sialidase-naive WT or $G D 35^{-/}$tissue cannot be simply ascribed to limited availability of GM1 ligand.

The heterogeneity of ganglioside and protein content in lipid rafts means there will be a wide range of raft-associated molecules that could potentially interact with GM1 or inhibit binding of anti-GM1 antibodies through steric hindrance. Our observation that cleavage of GPI-anchored proteins resulted in increased
DG2 and CBT binding suggests that these proteins have a general masking effect for GM1, although this was not the particular masking effect that prevented the binding of DG1, because that remained at the same low level.

Anti-GM1 mAbs cloned from neuropathy patients were also masked from binding GM1 and therefore unable to bind nerve terminals in ex vivo nerve-muscle preparations in these studies. This indicates that the observations with the mouse monoclonal antiGM1 antibody DG1 is likely to be clinically relevant in humans. Further studies are needed to explore the diversity of anti-GM1 antibodies seen in different patient populations to determine the extent of diversity in the context of ganglioside interaction-mediated inhibition. In recent experiments, we have identified antiGM1-positive neuropathy patient sera that are capable of inducing the neuropathophysiological effects at NMJs as observed here, but only after neuraminidase treatment (J.J. Plomp et al., unpublished observations). This further shows that clinically relevant anti-GM1 antisera exist that only bind to unmasked GM1. Such human antibodies may only become clinically important in conditions where the topological organization of gangliosides is destabilized, as for instance during nerve regeneration, and the mechanisms by which they may or may not induce immunopathology in normal nerve are currently unknown. In regenerating nerve fibers (following experimental transaction injury), it has been shown that growth cones have enhanced activity of a membrane sialidase that acts to reduce complex gangliosides into GM1 that may thereby render GM1 available for complex attenuated antibody binding. Targeted delivery of sialidase to experimental nerve lesions in the rat enhances axon outgrowth, and this may in part be a result of the increased level of GM1 in the regenerating axon (28). In addition, the sialidase-mediated decrease of GD1a interrupts the neuronal adhesion of myelin-associated glycoprotein, which normally inhibits regeneration (48), causing disinhibited axonal regeneration. Based on our observations with neuraminidase, it is possible that unmasking of cryptic GM1 could allow previously harmless DG1-like antibodies to bind to GM1-rich growth cones and therefore become pathogenic through their ability to impair regeneration. There is experimental evidence, at least for anti-GD1a antibodies, that antiganglioside antibodies may influence neuronal regeneration (49). The existence of anti-GM1 antibodies like DG1 that specifically bind regenerating neural tissue and cause complement-mediated injury would have significant clinical consequences in that such a phenomenon could be the start of recurrent cycles of axonal injury and regeneration. Therefore, rapid and persistent clearance of such antibodies should be an important therapeutic goal.

It is possible that GM1 masking occurs by interaction with gangliosides other than GD1a, and indeed for completely different ganglioside pairings. Indeed, in our control experiments, the Gal $(\beta 1-3)$ GalNAc moiety of GD1b was apparently shielded from binding DG2, whereas MOG1 - which presumably binds at least in part the internal disialosyl structure of GD1b as it is GD1b monospecific - was able to bind GD1b. Studies showing neuropathic effects restricted to sensory ataxia due to monospecific anti-GD1b antibodies in a rabbit model are consistent with this finding (50), as are recent studies using human ataxic GBS sera (51). We did not explore further the nature of this antibody-binding inhibitory interaction of GD1b with other gangliosides.

With regard to the induction of anti-GM1 antibodies by C. jejuni infection in GBS, our findings illuminate the protective role of antibody in microbial defence, as balanced against the develop- 
ment of autoimmune injury. Thus, DG1 is able to bind the GM1 mimic in LOS on live C. jejuni that also bears a GD1a mimic in a 50:50 ratio, indicating that a masking interaction does not occur. This shows that DG1-like antibodies could be bactericidal without binding to neuronal GM1 and thus (at least initially) would not exert an autoimmune neuropathic effect. Furthermore, DG1like-producing B cell clones do not recognize self-GM1 in living membranes. Therefore, breaking tolerance to GM1 might not be required in order to generate protective immunity against selfmimicking microbial molecules.

We were able to reproduce a masking effect of GD1a and other gangliosides on GM1 in immunoassays conducted either on polystyrene plates or PVDF membranes, recapitulating the discordant findings observed in living membranes between DG1 and DG2. Interestingly, the inhibitory complexes between GM1 and GD1a form on both polystyrene and PVDF immobilization platforms, and thus the molecular interactions between GM1 and other gangliosides must spontaneously form when they are admixed, in addition to being organized or disorganized in living or dead membranes, respectively. Furthermore, these solid-phase immunoassays demonstrated that interaction is not unique to GM1 and GD1a but can also occur between GM1 and other gangliosides. In addition, they form important support for the conclusion drawn from our neuraminidase experiments in live tissues - that the enabling factor for DG1 binding to the neuronal membrane under these conditions is an unmasking of preexisting GM1 by the removal of the external neuraminic acid of GD1a, as opposed to simply an increase in GM1 density by creation of de novo GM1 from GD1a.

Based on these experimental findings, we conclude that the microheterogeneity of ganglioside binding by anti-GM1 antibodies substantially influences their neuropathogenic potential and that the basis for these differences is likely due to subtle conformational arrangements within the membrane, leading to complexes of GM1 with other gangliosides that inhibit binding of some types of antiGM1 antibodies. These ganglioside interactions appear to be stably formed in living membranes but are highly susceptible to disintegration (and thus epitope exposure) in frozen or otherwise altered tissue. The changes in the molecular architecture of the living and dead/disrupted membrane that underlie these properties are unknown and difficult to investigate at the molecular biophysical level. In solid-phase immunoassays, these gangliosides interactions are also able to form. Recent studies showed that complexes of 2 different gangliosides can form neoepitopes that specifically bind certain neuropathy-associated antibodies that do not bind to the single ganglioside species $(5,52,53)$. Together with the data provided here, this leads to the conclusion that ganglioside interaction may either enable or inhibit antibody binding to the neuronal membrane or be neutral, depending of the type of circulating antibody. To describe these 3 conditions, we propose the terms complex-enhanced, complexattenuated, and complex-independent. In the light of these findings, we need to critically evaluate any individual antibody-ganglioside interaction detected by the wide range of techniques available in order to be able to interpret the neuropathogenic potency of such an interaction in the living neuronal membrane.

\section{Methods}

Anti-ganglioside $\mathrm{mAbs}$. Mice lacking complex gangliosides (GalNAcT $T^{-/}$mice) were used to generate anti-ganglioside antibodies in response to inoculation of ganglioside liposomes or ganglioside-mimicking LOS $(15,29,54)$. Hybridoma cells were maintained to generate stocks of mAbs, which were affinity purified by protein $\mathrm{G}$ sepharose chromatography (Amersham; GE Healthcare), desalted, and stored at $-70^{\circ} \mathrm{C}$. Their binding properties are shown in Table 1, calculated by half-maximal binding and BiaCore (GE Healthcare) studies. Half-maximal binding values represent the reciprocal of the antibody concentration (in $\mathrm{mg} / \mathrm{ml}$ ) giving half-maximal binding as determined by BiaCore using glycolipid-containing liposomes as the immobilized ligand and Fab fragments as the analyte, as previously reported (29). Human mAbs were cloned as previously described (38), and their binding properties are shown in Table 1.

Mice. Male and female GD3s $\mathrm{s}^{-/}$(30), GalNAcT $T^{-/}$(55), and WT mice were used at 4-8 weeks of age. All animal studies were approved by the committee on Animal Health and Care of Leiden University. Mice were humanely killed by administration of a rising concentration of $\mathrm{CO}_{2}$.

Immunohistochemistry on frozen tissue sections. For topical mAb binding studies, diaphragm was snap-frozen and cryostat sectioned at $8 \mu \mathrm{m}$ onto APES-coated (Sigma-Aldrich) slides. For staining, mAbs were applied at $20 \mu \mathrm{g} / \mathrm{ml}$ and CTB-FITC (Sigma-Aldrich) at $1 \mu \mathrm{g} / \mathrm{ml}$ diluted in PBS, along with $\alpha B$ Bx-TRITC $(1.3 \mu \mathrm{g} / \mathrm{ml}$; Invitrogen). $\mathrm{mAbs} / \mathrm{CTB}$ were incubated for 3.5 hours at $4^{\circ} \mathrm{C}$. For secondary detection of mAbs, slides were rinsed in PBS, and anti-mouse IgG-FITC $(1.3 \mu \mathrm{g} / \mathrm{ml}$ in PBS; SouthernBiotech) was applied for 3 hours at $4^{\circ} \mathrm{C}$. All slides were mounted in Citifluor (Citifluor Ltd.) following a final rinse in PBS. For CTB blocking of mAbs, sections were first incubated in unlabeled CTB $(4 \mu \mathrm{g} / \mathrm{ml}$ in PBS; Sigma-Aldrich) or PBS as a control, for 1.5 hours at $4{ }^{\circ} \mathrm{C}$, and rinsed thoroughly in PBS and mAbs applied as described above.

For Ig, C3, MAC, and neurofilament quantification, diaphragm (previously exposed ex vivo to mAbs and NHS as complement source) was cryostat cut at 8 and $20 \mu \mathrm{m}$ onto APES-coated slides (thicker sections were designated for neurofilament staining). All solutions were made up in PBS and incubations done at $4^{\circ} \mathrm{C}$. $\alpha$ BTx-TRITC was used at $1.3 \mu \mathrm{g} / \mathrm{ml}$ throughout. To detect $\mathrm{mAb}$ binding, FITC subtype-specific anti-mouse IgG or anti-human $\operatorname{IgM}$ (SouthernBiotech) was applied at $3.3 \mu \mathrm{g} / \mathrm{ml}$ for 3 hours at $4^{\circ} \mathrm{C}$, along with $\alpha$ BTx-TRITC. For detection of the activated complement component C3, slides were incubated in FITC-goat anti-human C3 (33 $\mu \mathrm{g} / \mathrm{ml}$; Dako) and $\alpha B T x$-TRITC for 2 hours at $4{ }^{\circ} \mathrm{C}$. MAC staining was performed with mouse anti-human C5b-9 (363 $\mu \mathrm{g} / \mathrm{ml}$; Dako), applied to slides for 3.5 hours with $\alpha B$ Tx-TRITC. After rinsing in PBS, MAC was detected with FITC-goat anti-mouse IgG2a applied at $5 \mu \mathrm{g} / \mathrm{ml}$ for 3.5 hours. Sections for neurofilament analysis were stained for 1 hour in $\alpha B T x-T R I T C$, rinsed in PBS, and placed for 20 minutes in freezing ethanol $\left(-20^{\circ} \mathrm{C}\right)$ prior to overnight application of 1211 (rabbit polyclonal, diluted 1:750; Affinity BioReagents). Slides were rinsed and incubated in FITC-goat anti-rabbit IgG $(3.3 \mu \mathrm{g} / \mathrm{ml})$ for 3.5 hours. Slides were finally rinsed and mounted in Citifluor.

Immunostaining of live tissue. For triangularis sternae neuromuscular preparations, the rib cage was removed and pinned in a Sylgard-lined (Sigma-Aldrich) dish with standard Ringer's medium (119 $\mathrm{mM} \mathrm{NaCl}$, $4.5 \mathrm{mM} \mathrm{KCl}_{2} 2 \mathrm{mM} \mathrm{CaCl}_{2}, 1 \mathrm{mM} \mathrm{MgSO} 4,1 \mathrm{mM} \mathrm{NaH} \mathrm{PO}_{4}, 23 \mathrm{mM}$ $\mathrm{NaHCO}_{3}, 11 \mathrm{mM}$ glucose, $\mathrm{pH} 7.4$, pre-gassed with $95 \% \mathrm{O}_{2} / 5 \% \mathrm{CO}_{2}$ ), and triangularis sternae was exposed. Preparations were incubated in $\mathrm{mAbs}$ $(100 \mu \mathrm{g} / \mathrm{ml}$ in Ringer's) or CTB $(2 \mu \mathrm{g} / \mathrm{ml})$ and $\alpha$ BTx-TRITC $(2 \mu \mathrm{g} / \mathrm{ml})$ for 2 hours at $32^{\circ} \mathrm{C}$, followed by 30 minutes at $4{ }^{\circ} \mathrm{C}$. Tissue was thoroughly rinsed in Ringer's and fixed for 20 minutes at room temperature in $4 \%$ PFA (Sigma-Aldrich). Tissue was rinsed again in Ringer's and incubated for 10 minutes in $0.1 \mathrm{M}$ glycine. For detection of mAbs, tissue was incubated overnight in subtype-specific FITC anti-mouse or anti-human IgM secondary antibodies $(3.3 \mu \mathrm{g} / \mathrm{ml}$ in PBS). For neurofilament staining, 1211 was applied overnight (rabbit polyclonal, diluted 1 in 200) in $1 \%$ Triton $\mathrm{X}-100 / \mathrm{PBS}$ and after 3 rinses in PBS, was detected with Cy5-anti-rabbit $\operatorname{IgG}(3.3 \mu \mathrm{g} / \mathrm{ml}$ in PBS $)$ at $4{ }^{\circ} \mathrm{C}$ for 8 hours. Following a final rinse in PBS, all immunostained muscles were whole mounted in Citifluor. 
Ex vivo pathogenesis: hemidiaphragm. Mouse hemidiaphragms with attached phrenic nerves were dissected out and pinned under tension in Sylgardlined dishes with oxygenated Ringer's medium. The dorsal portion of each was removed and immediately snap-frozen for baseline immunohistological measurements. Hemidiaphragms were incubated in either Ringer's (as a control) or mAbs $(100 \mu \mathrm{g} / \mathrm{ml})$ for 2 hours at $32^{\circ} \mathrm{C}$, followed by 30 minutes at $4^{\circ} \mathrm{C}$ and 10 minutes at room temperature. $\mathrm{mAb}$ solutions were removed and retained, and then preparations were rinsed in Ringer's.

NHS from a single donor stock was diluted to $40 \%$ in Ringer's and added as a source of complement. After 1 hour at room temperature, NHS was rinsed off and the diaphragm tissue snap-frozen before being processed for analysis.

Ex vivo electrophysiology at the NMJ. Left and right hemidiaphragms were dissected with their phrenic nerve attached and mounted in standard Ringer's medium (as above) at room temperature. mAbs and NHS were applied as described above.

Intracellular recordings of MEPPs at the NMJ were made from muscle fibers at room temperature $\left(20-22^{\circ} \mathrm{C}\right)$ by using a glass microelectrode (10-20 M $\Omega$, filled with $3 \mathrm{M} \mathrm{KCl}$ ) connected to a Geneclamp 500B (Axon Instruments; Molecular Devices) for amplifying and filtering (1 Hz highpass and $10 \mathrm{kHz}$ low-pass) of the signal. The signal was digitized using a Digidata 1322A interface (Axon Instruments; Molecular Devices) and analyzed using Mini Analysis 6.0.3 (Synaptosoft).

Electrophysiological data are presented as group mean \pm SEM of the mean muscle values calculated from the mean NMJ values. At least 10 NMJs were sampled per muscle per experimental condition.

Microscopy. For quantification of immunofluorescence over the NMJ, each experimental repeat ( $n$ of 3 animals/experiments for statistical analysis) was stained in a separate staining run, and at least 120 NMJs were imaged using constant acquisition settings for each marker. Dual-color images were acquired with a Zeiss LSM 5 Pascal microscope. All samples were analyzed by an observer blinded to the experimental protocol. Semiquantification was based on the $\alpha \mathrm{BT}$-TRITC signal to define the NMJ and was done with Scion Image analysis software (Scion Corp.), either by analyzing the percentage of immunofluorescence over the NMJ (as previously described; ref. 34) or analyzing the intensity of fluorescence over the NMJ. For illustrative images of whole-mount triangularis sternae, $3 \mathrm{D}$ reconstructions were generated from $z$-stacks using the software package Voxx-2 (http://www.nephrology.iupui.edu/imaging/voxx).

Membrane treatments. For ex vivo neuromuscular preparations, $1-5 \mu \mathrm{U} / \mathrm{ml}$ of neuraminidase (Clostridium perfringens; Sigma-Aldrich) was applied to tissue for $1-1.5$ hours at $32^{\circ} \mathrm{C}$. The enzyme was rinsed off and tissues incubated in $\mathrm{mAb}$ and/or complement as described above. For CTB preincubation of tissue prior to neuraminidase, unconjugated CTB $(4 \mu \mathrm{g} / \mathrm{ml})$ was applied to tissue for 1.5 hours at $32^{\circ} \mathrm{C}$ and thoroughly rinsed off with Ringer's prior to neuraminidase treatment. For GPI-anchored protein removal, PC12 cells were incubated at $37^{\circ} \mathrm{C}$ for 1 hour in $2 \mathrm{U} / \mathrm{ml}$ of PI-PLC (Sigma-Aldrich). Ex vivo neuromuscular preparations were treated for 1.5 hours at $32^{\circ} \mathrm{C}$ in $3.5 \mathrm{U}$ of PI-PLC. For cholesterol depletion, $5 \mathrm{mM}$ methyl- $\beta$-cyclodextrin (SigmaAldrich) was incubated for 45 minutes at $37^{\circ} \mathrm{C}$. Cellular cholesterol was measured using Infinity Cholesterol reagent (Alpha Laboratories).

GD1a/GM1 colocalization. MOG35 (IgG2b) was primary labeled using a Zenon Mouse IgG Labeling Kit (Invitrogen) according to the manufacturer's instructions. Colocalization of DG2 and MOG35 was determined using the Metamorph (version 6.3.3; Invitrogen) "correlation plot" application to compare the fluorescence in each pixel of the FITC and TRITC channels after subtracting background fluorescence.

PC12 cell staining. Cells were grown on poly-L-lysine-coated (SigmaAldrich) coverslips and mAbs applied $(12.5 \mu \mathrm{g} / \mathrm{ml}$ in PBS) for $30 \mathrm{~min}$ utes at $4^{\circ} \mathrm{C}$. Following 3 rinses in PBS, mAbs were detected with FITC anti-mouse $\operatorname{IgG}(3.3 \mu \mathrm{g} / \mathrm{ml})$ for 45 minutes at $4{ }^{\circ} \mathrm{C}$. Coverslips were rinsed again, fixed for 15 minutes in $4 \% \mathrm{PFA}$, and finally rinsed before mounting in Citifluor. For LIVE/DEAD staining (Invitrogen), a mixture of calcein green $(2 \mu \mathrm{M})$ and ethidium homodimer-1 (EthD-1, $2 \mu \mathrm{M})$ was applied to cells in PBS for 30 minutes at $37^{\circ} \mathrm{C}$. Coverslips were rinsed in PBS, mounted in PBS, and immediately imaged.

Raft isolation, affinity purification, and Western blotting. Approximately $1 \times 10^{8}$ PC1 2 cells were suspended in either MOG35 or control mAb EG1 (anti-GD3, -GT1a, and -GQ1b) at $15 \mu \mathrm{g} / \mathrm{ml}$ for 1 hour at $4^{\circ} \mathrm{C}$. Cells were washed in chilled PBS and kept on ice for subsequent steps. Cells were resuspended in $500 \mu \mathrm{l}$ of $25 \mathrm{mM}$ MES/150 mM NaCl, pH 6.5 (MBS), $0.5 \%$ Brij-96 (Sigma-Aldrich), and protease inhibitors (Roche Applied Science). Following incubation at $4{ }^{\circ} \mathrm{C}$ for 20 minutes, solubilized cells were homogenized with 10 strokes of a Dounce homogenizer. Of the lysate, $0.4 \mathrm{ml}$ was mixed with $80 \%$ sucrose and overlaid successively with $2.2 \mathrm{ml}$ of $30 \%$ sucrose and $1.4 \mathrm{ml}$ of $5 \%$ sucrose. Following overnight centrifugation at $240,000 \mathrm{~g}$, fraction 1 was collected by removing $390 \mu \mathrm{l}$ from the top of the gradient and successive fractions through to fraction 12 were collected in this way. Protease inhibitors were added immediately upon fraction collection.

Fractions 3-5 from MOG35-incubated cells were pooled following retention of an equal aliquot from each (for Western blotting along with other fractions). This was also done for control mAb-incubated cells. Pooled samples were halved: half was used for affinity purification and half retained as starting material. For affinity purification, anti-mouse IgG-coated Dynabeads (Invitrogen) were incubated with the samples for 30 minutes at $4{ }^{\circ} \mathrm{C}$ on a rotating mixer. Tubes were exposed to a magnet and the unbound material removed and retained. Beads were rinsed 4 times in PBS, Novex loading buffer (Invitrogen) was added to the rinsed beads, and the preparation was boiled to release the bound fractions for electrophoresis alongside unbound material and starting material.

For electrophoresis, samples were reduced by addition of $25 \mathrm{mM}$ DTT (Amersham; GE Healthcare) and run on Novex 16\% Tricene gels (Invitrogen). Gels were blotted overnight in a transfer cell according to the manufacturer's instructions (Bio-Rad) onto nitrocellulose membranes. Membranes were blocked in 5\% nonfat milk in PBS/0.01\% Tween-20 (PBST) and incubated in anti-ganglioside $\mathrm{mAbs}(20 \mu \mathrm{g} / \mathrm{ml})$ or $0.1 \mu \mathrm{g} / \mathrm{ml}$ HRP-conjugated CTB (Invitrogen). After rinsing, and if required, secondary antibody (HRP-conjugated anti-mouse IgG; Sigma-Aldrich) was added at $0.38 \mu \mathrm{g} / \mathrm{ml}$ in $1 \%$ nonfat milk/PBST. ECL (Amersham; GE Healthcare) was used to develop the membranes. Fractions 1-12 were also subject to Western blotting to confirm success of the raft isolation using antibodies against flotillin (BD), SNAP25 (56), and transferrin receptor (Invitrogen), all at $10 \mu \mathrm{g} / \mathrm{ml}$.

Immunoassays. Gangliosides were purchased from Sigma-Aldrich or Accurate. ELISA was performed as previously described (57). In brief, Immunolon $2 \mathrm{HB}$ plates (Thermo Scientific) were coated with $100 \mu \mathrm{l}$ of ganglioside $(2 \mu \mathrm{g} / \mathrm{ml})$ diluted in methanol. To create ganglioside complexes, a 50:50 (v/v) mixture of the 2 component gangliosides in methanol was sonicated for 3 minutes. A volume of $100 \mu$ l of this solution (containing $100 \mathrm{ng}$ of each ganglioside) was then applied to the appropriate wells. Control wells were coated with methanol alone. The average OD reading from control wells was subtracted from all other wells to correct for background. The mAbs DG1 and DG2, used at their GM1 half-maximal binding concentrations of $0.5 \mu \mathrm{g} / \mathrm{ml}$ and $0.4 \mu \mathrm{g} / \mathrm{ml}$, respectively, and the appropriate secondary antibodies (peroxidase-conjugated goat anti-mouse IgG; 1:3,000; Sigma-Aldrich) were diluted in $1 \%$ BSA. The assay was conducted at $4{ }^{\circ} \mathrm{C}$. For the PVDF glycoarray, gangliosides and ganglioside complexes $(100 \mu \mathrm{g} / \mathrm{ml})$ were prepared as described for ELISA. PVDF membrane on glass slides was spotted (100 nl per spot) with each 
target ganglioside or ganglioside complex in duplicate at predefined locations on each membrane using an automated TLC sampler (CAMAG) as previously described (58). Membranes were blocked with $2 \%$ BSA, then probed with the same primary $(1 \mu \mathrm{g} / \mathrm{ml})$ and secondary antibodies (diluted 1:30,000) as for ELISA. Chemiluminescence detection was performed using ECL plus (Amersham; GE Healthcare). Digitized film images were analyzed and quantified by the array analysis component of ImageQuant TL software (Amersham; GE Healthcare).

C. jejuni. Bacteria were cultured on Skirrow agar medium (E \& O Laboratories Ltd.) and maintained under microaerophilic conditions. For inactivation, bacteria were suspended in PBS and placed in a UV crosslinker for 30 minutes or suspended in 4\% PFA for 25 minutes.

FACS analysis. For FACS analysis, anti-ganglioside mAbs were used at $10 \mu \mathrm{g} / \mathrm{ml}$, CTB-FITC at $1 \mu \mathrm{g} / \mathrm{ml}$, and Thy-1 (CD90; Abcam) at $7.8 \mu \mathrm{g} / \mathrm{ml}$ in PBS. Secondary antibodies (anti-mouse IgG-FITC) were used at $3.3 \mu \mathrm{g} / \mathrm{ml}$, and cells were rinsed by pelleting in PBS. FACS analysis was performed using a FACScan flow cytometer (BD).

Statistics. The Mann-Whitney $U$ test was used for statistical analyses of nonparametric immunohistological data; otherwise, 1-way ANOVA was used to determine statistical significance, both employing a 5\% level of significance. Data are represented as box-and-whisker plots, with the horizontal line representing the median. One quarter of the data lying above the median and one quarter of the data lying below the median are represented by the large box, and the vertical lines on either side show the remaining quarters of the data lying above and below the interquartile ranges. For clarity, outliers are not shown, but they were included in the statistical analysis. To assess whether binding to GM1 complexes was statistically different from binding to GM1 alone on ELISA and PVDF array, general linear model ANOVA was used. Dunnett's method was employed to maintain a family error rate of less than 0.05 for multiple comparisons to the control level. Differences between DG1 and DG2 binding to each complex were assessed by the 2-tailed 2-sample $t$ test. Bonferroni's correction was applied in view of the multiple comparisons being made, again to maintain a family error rate of less than 0.05 . The normality of each data set was verified by the Kolmogorov-Smirnov test (with $P>0.15$ indicating normality).

\section{Acknowledgments}

This work was sponsored by grants from the Wellcome Trust (077041/Z/05/Z to H.J. Willison) and the Prinses Beatrix Fonds (MAR04-0213 to J.J. Plomp). K.N. Greenshields was supported by the Medical Research Council doctoral training account awarded to the University of Glasgow.

Received for publication September 2, 2008, and accepted in revised form December 22, 2008.

Address correspondence to: Hugh J. Willison, Glasgow Biomedical Research Centre, Room B330, 120 University Place, University of Glasgow, Glasgow G12 8TA, United Kingdom. Phone: 44-141-330-8384; Fax: 44-141-201-2993; E-mail: h.j.willison@ clinmed.gla.ac.uk.
1. Sandhoff, K., and Kolter, T. 2003. Biosynthesis and degradation of mammalian glycosphingolipids. Philos. Trans. R. Soc. Lond. B Biol. Sci. 358:847-861.

2. Simons, K., and Ikonen, E. 1997. Functional rafts in cell membranes. Nature. 387:569-572.

3. Varki, A. 2007. Glycan-based interactions involving vertebrate sialic-acid-recognizing proteins. Nature. 446:1023-1029.

4. Bullens, R.W., et al. 2002. Complex gangliosides at the neuromuscular junction are membrane receptors for autoantibodies and botulinum neurotoxin but redundant for normal synaptic function. J. Neurosci. 22:6876-6884.

5. Willison, H.J. 2005. Ganglioside complexes: new autoantibody targets in Guillain-Barre syndromes. Nat. Clin. Pract. Neurol. 1:2-3.

6. Crocker, P.R., Paulson, J.C., and Varki, A. 2007. Siglecs and their roles in the immune system. Nat. Rev. Immunol. 7:255-266.

7. Lencer, W.I., and Tsai, B. 2003. The intracellular voyage of cholera toxin: going retro. Trends Biochem. Sci. 28:639-645.

8. Tsai, B., et al. 2003. Gangliosides are receptors for murine polyoma virus and SV40. EMBO J 22:4346-4355.

9. Willison, H.J., and Yuki, N. 2002. Peripheral neuropathies and anti-glycolipid antibodies. Brain. 125:2591-2625.

10. Yuki, N. 2001. Infectious origins of, and molecular mimicry in, Guillain-Barre and Fisher syndromes. Lancet Infect. Dis. 1:29-37.

11. Goodfellow, J.A., et al. 2005. Overexpression of GD1a ganglioside sensitizes motor nerve terminals to anti-GD1a antibody-mediated injury in a model of acute motor axonal neuropathy. J. Neurosci. 25:1620-1628.

12. Kusunoki, S., et al. 1996. Experimental sensory neuropathy induced by sensitization with ganglioside GD1b. Ann. Neurol. 39:424-431.

13. Yuki, N., et al. 2001. Animal model of axonal Guillain-Barre syndrome induced by sensitization with GM1 ganglioside. Ann. Neurol. 49:712-720.

14. Plomp, J.J., et al. 1999. Miller Fisher anti-GQ1b anti- bodies: a-latrotoxin-like effects on motor end plates. Ann. Neurol. 45:189-199.

15. Bowes, T., et al. 2002. Tolerance to self gangliosides is the major factor restricting the antibody response to lipopolysaccharide core oligosaccharides in Campylobacter jejuni strains associated with Guillain-Barre syndrome. Infect. Immun. 70:5008-5018.

16. Yuki, N., et al. 1993. A bacterium lipopolysaccharide that elicits Guillain-Barre syndrome has a GM1 ganglioside-like structure. J. Exp. Med. 178:1771-1775.

17. Yuki, N., et al. 2004. Carbohydrate mimicry between human ganglioside GM1 and Campylobacter jejuni lipooligosaccharide causes Guillain-Barre syndrome. Proc. Natl. Acad. Sci. U. S. A. 101:11404-11409.

18. Ang, C.W., et al. 2002. Structure of Campylobacter jejuni lipopolysaccharides determines antiganglioside specificity and clinical features of GuillainBarre and Miller Fisher patients. Infect. Immun. 70:1202-1208

19. Arasaki, K., Kusunoki, S., Kudo, N., and Kanazawa, I. 1993. Acute conduction block in vitro following exposure to antiganglioside sera. Muscle Nerve. 16:587-593.

20. Buchwald, B., et al. 2007. Anti-ganglioside antibodies alter presynaptic release and calcium influx. Neurobiol. Dis. 28:113-121.

21. Nores, G.A., et al. 2007. Anti-GM1 antibodies as a model of the immune response to self-glycans. Biochim. Biophys. Acta. 1780:538-545.

22. Paparounas, K., O'Hanlon, G.M., O'Leary, C.P., Rowan, E.G., and Willison, H.J. 1999. Anti-ganglioside antibodies can bind peripheral nerve nodes of Ranvier and activate the complement cascade without inducing acute conduction block in vitro. Brain. 122:807-816.

23. Schwarz, A., and Futerman, A.H. 1996. The localization of gangliosides in neurons of the central nervous system: the use of anti-ganglioside antibodies. Biochim. Biophys. Acta. 1286:247-267.

24. Iwamoto, N., et al. 1990. Cell membrane changes in brains manifesting senile plaques: an immunohistochemical study of GM1 membranous ganglioside. Brain Res. 522:152-156.

25. Laev, H., and Mahadik, S.P. 1989. Topography of monosialoganglioside (GM1) in rat brain using monoclonal antibodies. Neurosci. Lett. 102:7-14.

26. Goodyear, C.S., et al. 1999. Monoclonal antibodies raised against Guillain-Barre syndrome-associated Campylobacter jejuni lipopolysaccharides react with neuronal gangliosides and paralyze musclenerve preparations. J. Clin. Invest. 104:697-708.

27. Halstead, S.K., et al. 2004. Anti-disialoside antibodies kill perisynaptic Schwann cells and damage motor nerve terminals via membrane attack complex in a murine model of neuropathy. Brain. 127:2109-2123.

28. Yang, L.J., et al. 2006. Sialidase enhances spinal axon outgrowth in vivo. Proc. Natl. Acad. Sci. U. S. A. 103:11057-11062.

29. Townson, K., et al. 2007. Solid phase immunoadsorption for therapeutic and analytical studies on neuropathy-associated anti-GM1 antibodies. Glycobiology. 17:294-303.

30. Okada, M., et al. 2002. b-series Ganglioside deficiency exhibits no definite changes in the neurogenesis and the sensitivity to Fas-mediated apoptosis but impairs regeneration of the lesioned hypoglossal nerve. J. Biol. Chem. 277:1633-1636.

31. Merritt, E.A., et al. 1994. Crystal structure of cholera toxin B-pentamer bound to receptor GM1 pentasaccharide. Protein Sci. 3:166-175.

32. Court, F.A., et al. 2008. Identity, developmental restriction and reactivity of extralaminar cells capping mammalian neuromuscular junctions. J. Cell. Sci. 121:3901-3911.

33. Goodyear, C.S., et al. 1999. Monoclonal antibodies raised against Guillain Barré syndrome-associated Campylobacter jejuni lipopolysaccharides react with neuronal gangliosides and paralyse nerve muscle preparations. J. Clin. Invest. 104:697-708.

34. O'Hanlon, G.M., et al. 2001. Anti-GQ1b ganglioside antibodies mediate complement-dependent destruction of the motor nerve terminal. Brain. 124:893-906 
35. Boffey, J., et al. 2005. Characterisation of the immunoglobulin variable region gene usage encoding the murine anti-ganglioside antibody repertoire. J. Neuroimmunol. 165:92-103.

36. Seyfried, T.N., el Abbadi, M., Ecsedy, J.A., Bai, H.W and Yohe, H.C. 1996. Influence of host cell infiltration on the glycolipid content of mouse brain tumors. J. Neurochem. 66:2026-2033.

37. Townson, K.H., et al. 2008. Glycosphingolipid depletion in PC12 cells using iminosugars protects neuronal membranes from anti-ganglioside antibody mediated injury. J. Neuroimmunol. 203:33-38.

38. Paterson, G., Wilson, G., Kennedy, P.G.E., and Willison, H.J. 1995. Analysis of anti-GM1 ganglioside IgM antibodies cloned from motor neuropathy patients demonstrates diverse v-region gene usage with extensive somatic mutation. J. Immunol. 155:3049-3059.

39. Ho, T.W., et al. 1997. Motor nerve terminal degeneration provides a potential mechanism for rapid recovery in acute motor axonal neuropathy after Campylobacter infection. Neurology. 48:717-724.

40. Susuki, K., et al. 2003. Acute motor axonal neuropathy rabbit model: immune attack on nerve root axons. Ann. Neurol. 54:383-388.

41. Kuziemko, G.M., Stroh, M., and Stevens, R.C. 1996. Cholera toxin binding affinity and specificity for gangliosides determined by surface plasmon resonance. Biochemistry. 35:6375-6384.

42. Paulson, J.C., Blixt, O., and Collins, B.E. 2006 Sweet spots in functional glycomics. Nat. Chem.
Biol. 2:238-248

43. Hakomori, S.I., Teather, C., and Andrews, H. 1968 Organizational difference of cell surface "hematoside" in normal and virally transformed cells. Biochem. Biophys. Res. Commun. 33:563-568.

44. Lloyd, K.O., Gordon, C.M., Thampoe, I.J., and DiBenedetto, C. 1992. Cell surface accessibility of individual gangliosides in malignant melanoma cells to antibodies is influenced by the total ganglioside composition of the cells. Cancer Res. 52:4948-4953.

45. Iwabuchi, K., Handa, K., and Hakomori, S. 1998. Separation of "glycosphingolipid signaling domain" from caveolin-containing membrane fraction in mouse melanoma B16 cells and its role in cell adhesion coupled with signaling. J. Biol. Chem. 273:33766-33773.

46. Hakomori, S.I. 2002. Inaugural article: the glycosynapse. Proc. Natl. Acad. Sci. U. S. A. 99:225-232.

47. Sonnino, S., Mauri, L., Chigorno, V., and Prinetti, A. 2007. Gangliosides as components of lipid membrane domains. Glycobiology. 17:1R-13R.

48. Vyas, A.A., et al. 2002. Gangliosides are functional nerve cell ligands for myelin-associated glycoprotein (MAG), an inhibitor of nerve regeneration. Proc. Natl. Acad. Sci. U. S. A. 99:8412-8417.

49. Lehmann, H.C., et al. 2007. Passive immunization with anti-ganglioside antibodies directly inhibits axon regeneration in an animal model. J. Neurosci. 27:27-34.

50. Kusunoki, S., Hitoshi, S., Kaida, K., Arita, M., and
Kanazawa, I. 1999. Monospecific anti-GD1b IgG is required to induce rabbit ataxic neuropathy. Ann. Neurol. 45:400-403.

51. Kaida, K., et al. 2008. GD1b-specific antibody induces ataxia in Guillain-Barre syndrome. Neurology. 71:196-201.

52. Kaida, K., et al. 2004. Ganglioside complexes as new target antigens in Guillain-Barre syndrome. Ann. Neurol. 56:567-571.

53. Kaida, K.I., et al. 2006. Anti-ganglioside complex antibodies in Miller Fisher syndrome. J. Neurol. Neurosurg. Psychiatry. 77:1043-1046.

54. Aspinall, G.O., et al. 1994. Lipopolysaccharides from Campylobacter-jejuni associated with Guillain-Barre-syndrome patients mimic human gangliosides in structure. Infect. Immun. 62:2122-2125.

55. Takamiya, K., et al. 1996. Mice with disrupted GM2/ GD2 synthase gene lack complex gangliosides but exhibit only subtle defects in their nervous system. Proc. Natl. Acad. Sci. U. S. A. 93:10662-10667.

56. Chamberlain, L.H., Burgoyne, R.D., and Gould, G.W. 2001. SNARE proteins are highly enriched in lipid rafts in PC12 cells: implications for the spatial control of exocytosis. Proc. Natl. Acad. Sci. U. S. A. 98:5619-5624.

57. Willison, H.J., et al. 1999. Inter-laboratory validation of an ELISA for the determination of serum anti-ganglioside antibodies. Eur. J. Neurol. 6:71-77.

58. Kanter, J.L., et al. 2006. Lipid microarrays identify key mediators of autoimmune brain inflammation. Nat. Med. 12:138-143. 